TRANSACTIONS OF THE

AMERICAN MATHEMATICAL SOCIETY

Volume 177, March 1973

\title{
ISOMORPHISM OF SIMPLE LIE ALGEBRAS
}

BY

\section{B. N. ALLISON}

ABSTRACT. Let $\mathfrak{L}$ and $\mathfrak{L}^{\prime}$ be finite dimensional simple Lie algebras over a field of characteristic zero. A necessary and sufficient condition is given for $\mathcal{L}$ and $\mathcal{L}^{\prime}$ to be isomorphic. The ani sotropic kernel of $\mathcal{L}$ is also studied. In particular, a result about this kernel in the rank one reduced case is proved. This result is then used to prove a conjugacy theorem for the simple summands of the anisotropic kernel in the general reduced case. The results and methods of this paper are rational in the sense that they involve no extension of the base field.

0 . Introduction. Let $\mathscr{L}$ and $\mathfrak{Q}^{\prime}$ be simple Lie algebras over a field $\Phi$ of characteristic zero. In this paper, we study the problem of determining when $\mathscr{L}$ and $\mathcal{L}^{\prime}$ are isomorphic. One approach to this problem has been taken by Satake in [3] and Tits in [6]. (They actually studied the corresponding problem for algebraic groups. See Seligman's [4] for their results in the Lie algebra case.) Their method involves extending $\Phi$ to a splitting field $\Omega$ for $\mathcal{L}$ and $\mathcal{L}^{\prime}$ and then using the classical criterion for isomorphism of $\mathcal{L}_{\Omega}$ and $\mathfrak{L}_{\boldsymbol{\Omega}}^{\prime}$. The main theorem of Tits and Satake is nonrational in the sense that both the statement and the proof of the theorem involve the extension field $\Omega / \Phi$ in an essential way. Our main theorem (9.1) on the other hand gives rational criteria for is omorphism of $\mathcal{L}$ and $\mathcal{L}^{\prime}$. The proof of 9.1 is also rational and makes no use of the Tits-Satake result.

$\$ \$ 1$ through 5 provide us with the tools which will be used in our study of the isomorphism question. In $\$ 6$, we give rational proofs of certain results about the anisotropic kernel in the reduced case. In particular, we prove a conjugacy result for the simple summands of this kernel. The basic tool here is the study of the rank 1 case. In $\$ \$ 7$ and 8 , we return to the preparation for the proof of 9.1 . In $\S 9$, we state and prove the isomorphism result.

In the succeeding sections, we will introduce notation for certain objects associated with $\mathcal{L}$. It will be assumed without comment that the corresponding primed notation has been introduced for $\mathcal{L}^{\prime}$.

Received by the editors January 25, 1972.

AMS (MOS) subject classifications (1970). Primary 17B05, 17B20, 17B40; Secondary $17 \mathrm{~B} 10,17 \mathrm{~B} 25$.

Key words and phrases. Isomorphism of Lie algebras, simple Lie algebras, anisotropic kernel, rank one Lie algebras, equivalence of representations, centrum, root systems, Weyl group, subalgebras of Lie algebras. 
1. Preliminary facts about $\mathscr{L}$. In this section, we introduce certain fundamental notions about the simple algebra $\mathcal{L}$. Proofs for all the results of this section can be found in the first three sections of [4].

1.1. A split toral subalgebra of $\mathcal{Q}$ is a commutative subalgebra $\mathcal{K}$ such that $\operatorname{ad}_{\mathfrak{Q}}(K)$ is semisimple with characteristic roots in $\Phi$ for all $K \in \mathcal{K}$. We assume $\mathfrak{T}$ is a maximal split toral subalgebra of $\mathcal{L}$. Any two such subalgebras are conjugate under the automorphism group of $\mathfrak{L}$. The dimension $r$ of $\mathfrak{T}$ is called the rank of $\mathscr{L}$.

1.2. We may write $\mathfrak{L}=\bigoplus \Sigma_{\gamma} \mathfrak{L}$, where $\gamma$ runs over all elements of the dual space $\mathfrak{I}^{*}$ of $\mathfrak{T}$ and $\mathfrak{L}_{\gamma}=\{L \in \mathfrak{L}:[L, T]=\gamma(T) L$ for all $T \in \mathfrak{T}\}$. The $\gamma \in \mathfrak{T}^{*}$ for which $\mathscr{L}_{\gamma} \neq(0)$ are called the roots of $\mathfrak{T}$. Let $\Sigma$ be the set of nonzero roots of $\mathcal{T}$. Then,

$$
\mathfrak{L}=\mathfrak{L}_{0} \oplus \sum_{\gamma \in \mathbf{\Sigma}} \mathfrak{L}_{\gamma},
$$

where $\mathscr{L}_{0}$ is the centralizer of $\mathfrak{T}$ in $\mathscr{L}$ and hence $\mathfrak{T} \subseteq \mathfrak{L}_{0}$.

1.3. Let $\mathcal{C}$ be the center of $\mathscr{L}_{0}$. Then, $\mathcal{T} \subseteq \mathcal{C}$ and $\mathscr{L}_{0}=\mathcal{C} \oplus\left[\mathscr{L}_{0}, \mathscr{L}_{0}\right]$, where $\left[\mathfrak{L}_{0}, \mathfrak{L}_{0}\right]$ is semisimple. $\left[\mathfrak{L}_{0}, \mathfrak{L}_{0}\right]$ is called the anisotropic kernel of $\mathscr{L}_{\text {. If }} \mathfrak{L}_{0}=$ $\mathcal{T}$, we say $\mathscr{L}$ is split. In the split case, $\mathcal{T}$ is a Cartan subalgebra of $\mathscr{L}$. In any case, $\mathcal{T}$ is always contained in a Cartan subalgebra of $\mathscr{Q}$. If $\mathcal{T}=(0), \mathfrak{L}$ is said to be anisotropic.

1.4. We have $\left[\mathfrak{L}_{\gamma}, \mathfrak{L}_{\delta}\right] \subseteq \mathfrak{Q}_{\gamma+\delta}$ for $\gamma, \delta \in \mathcal{T}^{*}$. Thus, if $\gamma \in \Sigma$, we have $\left[L_{\gamma}\right.$, $\left.L_{0}\right] \subseteq \mathfrak{L}_{\gamma}$ and hence $\mathscr{L}_{\gamma}$ is an $\mathfrak{L}_{0}$-module under the adjoint action.' In fact, $\mathfrak{L}_{\gamma}$ is an irreducible $\mathfrak{L}_{0}$-module. We denote the corresponding representation by $\mathcal{L}_{0}{ }^{\rho} \underline{\underline{\gamma}}$ End $_{\Phi}\left(\varrho_{\gamma}\right)$. If $\gamma, \delta, \gamma+\delta \in \Sigma$, we have $\left[\complement_{\gamma}, \mathfrak{\complement}_{\delta}\right] \neq(0)$, and hence, by irreducibility, $\left[\mathfrak{L}_{\gamma}, \mathfrak{L}_{\delta}\right]=\mathfrak{L}_{\gamma+\delta}$.

1.5. We denote the Killing form of $\mathcal{L}$ by $($,$) . This form when restricted to$ $\mathfrak{L}_{0}, \mathfrak{T}, \mathcal{C}$, or $\left[\mathscr{C}_{0}, \mathfrak{L}_{0}\right]$ is nondegenerate. We obtain a corresponding form $($,$) on$ the dual space $\mathcal{T}^{*}$ in the usual way. Let $\chi_{\mathbf{\Sigma}}$ be the $\mathbf{Q}$-subspace of $\mathcal{T}^{*}$ generated by $\Sigma$. Then, $\chi_{\Sigma}$ generates $\mathcal{I}^{*}$ as a $\Phi$-space and $\chi_{\Sigma}$ has dimension $r$ over $Q$. The form $\left(\right.$, ) restricts to a positive definite form on $\chi_{\Sigma}$ relative to which $\Sigma$ is a root system, i.e., if $\gamma, \delta \in \Sigma$, then $2(\gamma, \delta) /(\delta, \delta)$ is an integer and $\gamma-j \delta \in \Sigma \cup\{0\}$ for every integer $j$ between 0 and $2(\gamma, \delta) /(\delta, \delta)$. We may choose a fundamental system $\Pi$ for $\Sigma$, i.e. a linearly independent subset $\Pi$ of $\Sigma$ such that every element of $\Sigma$ is a positive or negative integral combination of elements of $\Pi . \Sigma^{+}$(resp. $\Sigma^{-}$) is defined to be the set of positive (resp. negative) roots relative to $\Pi$.

1.6 If $\gamma \in \Sigma$ and $k \gamma \in \Sigma$ for some $k \in \Phi$, then $k= \pm 1 / 2, \pm 1$, or \pm 2 . If $2 \gamma k \Sigma$ for all $\gamma \in \Sigma$, we say $\mathcal{L}$ is reduced.

1.7 We may associate a Dynkin diagram to $\Pi$ in the usual way with the following additional convention: If $\gamma \in \Pi$ and $2 \gamma \in \Sigma$, we place a square around the point of the diagram corresponding to $\gamma$. If $\mathcal{Q}$ is reduced, only the usual possibilities exist for the Dynkin diagram. If $\mathcal{Q}$ is not reduced, the Dynkin diagram is either 


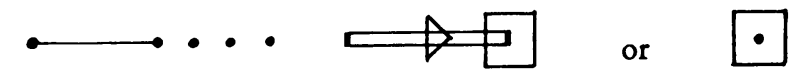

(the first possibility exists when $r>1$ and the second when $r=1$ ). In either case, we say $\mathcal{L}$ is of the type $B C_{r}$.

1.8. For $\gamma \in \Sigma$, define $\chi_{\Sigma} \stackrel{\omega^{2}}{\rightarrow} \quad \chi_{\Sigma}$ by $\epsilon^{\omega^{2}} \gamma=\epsilon-2(\epsilon, \gamma) /(\gamma, \gamma)$ for $\epsilon \epsilon \chi_{\Sigma}$. The group $\left\{\right.$ generated by $\left\{\omega_{\gamma}\right\}_{\gamma \in \Sigma}$ is called the Weyl group of $\mathcal{L}$. W is generated by $\left\{w_{\gamma}\right\}_{\gamma \in \Pi}$.

1.9. Suppose $\gamma \in \Sigma$. Then, there exists a unique element $T_{\gamma}$ of $\mathfrak{T}$ such that $T_{\gamma} \in\left[\mathscr{\complement}_{\gamma}, \mathfrak{L}_{-\gamma}\right]$ and $\gamma\left(T_{\gamma}\right)=2$. Moreover, for $X_{\gamma} \neq 0$ in $\mathfrak{\complement}_{\gamma}$, there exists $Y_{\gamma} \neq 0$ in $\mathcal{L}_{-\gamma}$ such that $\left[X_{\gamma}, Y_{\gamma}\right]=T_{\gamma}$.

Putting $\delta_{\gamma}=\Phi X_{\gamma} \oplus \Phi T_{\gamma} \oplus \Phi Y_{\gamma}, \delta_{\gamma}$ is a three dimensional split simple Lie algebra with multiplication table $\left[X_{\gamma}, Y_{\gamma}\right]=T_{\gamma},\left[X_{\gamma}, T_{\gamma}\right]=2 X_{\gamma},\left[Y_{\gamma}, T_{\gamma}\right]=$ $-2 Y_{\gamma}$. The representation theory for $\mathcal{S}_{\gamma}$ is well known (see for example p. 83 of [1] or p. 6 of [4]) and will be used without comment.

1.10. $\left\{T_{\gamma}\right\}_{\gamma \in \Pi}$ is a basis for $\mathcal{T}$ and hence $\mathcal{T}$ has a basis consisting of elements $T$ such that $\operatorname{ad}_{\mathfrak{Q}}(T)$ has integral characteristic roots.

1.11. Lemma. Suppose $\stackrel{\rho}{\rightarrow}$ End $_{\Phi}(\mathcal{O})$ is a representation of $\mathcal{L}$. Then, $L_{0}^{\rho}$ is semisimple and $T^{\rho}$ is semisimple with characteristic roots in $\Phi$ for $L_{0} \in \mathscr{L}_{0}$ and $T \in \mathcal{J}$.

Proof. For $\gamma \in \Sigma$, it follows from the representation theory for $\mathcal{S}_{\gamma}$ that $T_{\gamma}^{\rho}$ is semisimple with characteristic roots in $\Phi$. Thus $T^{\rho}$ has the same property for $T \in \mathfrak{T}$. Since $\mathcal{L}_{0}^{\rho}$ is completely reducible (by Theorem 3.18 of [1]), it remains only to show that if $E \in \mathscr{Q}_{0}^{\rho}$ is nilpotent, then $E=0$. Suppose $E \neq 0$. By Theorem 3.17 of $[1]$, there exist nonzero $H, F \in \mathfrak{L}_{0}^{\rho}$ such that $[E, F]=H,[E, H]=2 E$, and $[F, H]=-2 F$. From the representation theory for $\Phi E \oplus \Phi H \oplus \Phi F$, it follows that $\operatorname{ad}_{\rho} \rho(H)$ is semisimple with characteristic roots in $\Phi$. Hence, $H \in \mathcal{T}^{\rho}$. But $E$ and $H$ do not commute, giving a contradiction.

1.12. We define $G$ to be the group of automorphisms $\phi$ of $\mathcal{Q}$ such that $\mathfrak{T}^{\phi}=\mathfrak{J}$ and $\phi=\exp \left(\operatorname{ad}\left(L_{1}\right)\right) \cdots \exp \left(\operatorname{ad}\left(L_{k}\right)\right)$ for some $L_{1}, L_{2}, \cdots, L_{k} \in \mathfrak{\&}$ such that $\operatorname{ad}\left(L_{1}\right)$, $\cdots, \operatorname{ad}\left(L_{k}\right)$ are nilpotent.

2. Reductive algebras and inner automorphisms.

2.1. We recall that a reductive $\mathrm{Lie}$ algebra is a Lie algebra $M$ such that $M=$ center $(\mathbb{M}) \oplus[\mathbb{M}, \mathbb{M}]$ and $[\mathbb{M}, \mathbb{M}]$ is semisimple. In particular, $\mathfrak{L}_{0}$ is reductive. The following lemma is well known:

2.2. Lemma. If $\mathcal{G}$ is an ideal of a reductive Lie algebra $M$, then $\mathscr{I}=(\mathrm{I} \cap$ center $(\pi)) \oplus(9 \cap[\pi, \pi])$.

2.3. Let $M$ be a reductive Lie algebra. An automorphism $\psi$ of $M$ is said to be 
inner if there exists a finite extension $\Omega / \Phi$ and elements $M_{1}, \ldots, M_{k} \in M_{\Omega}$ such that $\operatorname{ad}\left(M_{1}\right), \cdots, \operatorname{ad}\left(M_{k}\right)$ are nilpotent and $\phi=\exp \left(\operatorname{ad}\left(M_{1}\right)\right) \cdots \exp \left(\operatorname{ad}\left(M_{k}\right)\right)$.

2.4. Lemma. Let $\psi$ be an inner automorphism of a reductive Lie algebra $\mathbb{M}$. Then,

(i) $\psi$ commutes with the centrum of $\mathbb{M}$,

(ii) $\psi$ fixes the elements of center(M),

(iii) $\psi$ stabilizes each ideal of $\mathbb{N}$,

(iv) If $M \stackrel{\rho}{\rightarrow} \operatorname{End}_{\boldsymbol{\Phi}}(0)$ is a representation of $M$ such that $M^{\rho}$ generates End $_{\boldsymbol{\Phi}}(0)$ as an associative algebra with 1 , then there exists an invertible element $\eta$ of End $_{\Phi}\left(\mathcal{C}^{\eta}\right)$ such that $\eta^{-1} M^{\rho} \eta=\left(M^{\psi}\right)^{\rho}$ for $M \in M$.

Proof. (i), (ii) and (iii) are clear. To prove (iv), choose $\Omega / \Phi$ and $M_{1}, \cdots, M_{k} \epsilon$ $M_{\Omega}$ as in 2.3. We may assume $M_{1}, \ldots, M_{k} \in\left[\pi, M_{\Omega}\right.$ and $\Omega / \Phi$ is Galois with Galois group $\mathfrak{G}_{\text {. Since }} \operatorname{ad}[\pi, \pi]_{\Omega}\left(M_{i}\right)$ is nilpotent, $M_{i}^{\rho}$ is nilpotent, $i=1, \cdots, k$. Moreover, one easily checks that if $\xi=\exp \left(M_{1}^{\rho}\right) \cdots \exp \left(M_{k}^{\rho}\right)$, then $\left(M^{\psi}\right)^{\rho}=\xi^{-1} M^{\rho} \xi$ for $M \in \mathbb{M}$. Then, applying Hilbert's Theorem 90 , it is easy to see that $a \xi \epsilon$ $\operatorname{End}_{\Phi}(\mathcal{O})$ for some $a \in \Omega^{*}$. Put $\eta=a \xi$.

2.5. Lemma. Suppose $\psi \in G$ fixes the elements of $\mathcal{T}$. Then $\psi||_{0}$ is inner.

Proof. Write $\psi=\psi_{s} \psi_{u}$, where $\psi_{s}$ is semisimple and $\psi_{u}$ is unipotent. Then,

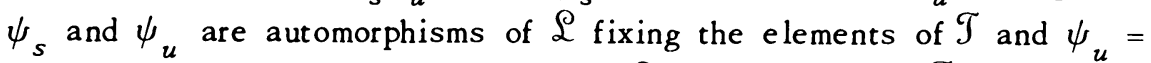
$\exp \left(\log \left(\psi_{u}\right)\right) \cdot \log \left(\psi_{u}\right)$ is a derivation of $\mathfrak{Q}$ which is zero on $\mathfrak{T}$. Hence, $\log \left(\psi_{u}\right)=$ $\operatorname{ad}(L)$ for some $L \in \mathscr{Q}_{0}$. Thus, $\operatorname{ad}(L)$ is both nilpotent and semisimple (by 1.11). Therefore, $\operatorname{ad}(L)=0$ and hence $\psi=\psi_{s}$ is semisimple. Let $\mathfrak{Q}_{1}$ be the set of elements of $\mathscr{Q}$ fixed by $\psi$. If $\Omega$ is the algebraic closure of $\Phi,\left(\mathscr{C}_{1}\right)_{\Omega}$ contains a Cartan subalgebra of $\left(\mathscr{C}_{\Omega}\right.$ (by Theorem 7 of [2]). But then since $\mathscr{L}_{1}$ is reductive (Theorem 1 of [2]), any Cartan subalgebra of $\mathcal{L}_{1}$ is a Cartan subalgebra of $\mathcal{Q}$. However, one such Cartan subalgebra $\mathcal{H}$ can be chosen containing $\mathcal{T}$. Thus, there exists a Cartan subalgebra $\mathcal{H}$ of $\mathfrak{L}$ containing $\mathfrak{T}$ and fixed by $\psi$. Since $\mathcal{H}$ is abelian, $\mathcal{H} \subseteq \mathfrak{L}_{0}$. But then $\mathcal{H}=\mathcal{C} \oplus\left(\mathcal{H} \cap\left[\mathfrak{C}_{0}, \mathfrak{L}_{0}\right]\right)$. Thus, $\psi$ fixes the elements of $\mathcal{C}$ and the elements of a Cartan subalgebra of $\left[\complement_{0}, \complement_{0}\right]$. This implies that $\psi \mid \complement_{0}$ is inner.

3. Subalgebras of $\mathscr{Q}$. Suppose $P$ is a nonempty subset of $\Sigma$ such that $\gamma-\delta \notin \Sigma$ for $\gamma, \delta \in P$ and $P^{\mathcal{N}} \subseteq \Sigma^{+}$for some $w \in$ W. Let $\chi_{P}$ be the Q-subspace of $\chi_{\Sigma}$ generated by $P$. Let $\langle P\rangle$ (resp. $\langle P\rangle^{+} ;\langle P\rangle-$ ) be the set of $\gamma \in \Sigma$ which are integral (resp. positive integral; negative integral) combinations of elements of $P$.

3.1. Lemma. $\langle P\rangle$ is a root system in $\chi_{P}$ with fundamental system $P$. Hence, $\langle P\rangle=\langle P\rangle^{+} \cup\langle P\rangle-$ and $\langle P\rangle^{+} \cap\langle P\rangle-=\varnothing$.

Proof. In this proof, $n_{\gamma}$ and $n_{\delta}$ denote nonnegative integers. $\langle P\rangle$ is clearly a root system. $P$ is linearly independent by Lemma 4 of [5]. To complete the 
proof, we must show that if $\Sigma_{n_{\gamma}} \geq 1$ and $\Sigma n_{\delta} \geq 1$, then $\Sigma_{n_{\gamma}} \gamma-\Sigma_{n_{\delta}} \delta \notin \Sigma$, where $\gamma$ and $\delta$ run over disjoint parts of $P$. This however follows from an easy induction on $\Sigma n_{\gamma}+\Sigma n_{\delta}$.

3.2. Put $\mathfrak{L}_{0}^{P}=\Sigma_{\gamma \in\langle P\rangle}\left[\mathfrak{L}_{\gamma}, \mathfrak{L}_{-\gamma}\right], \mathfrak{L}^{P}=\mathfrak{L}_{0}^{P} \oplus\left(\bigoplus \Sigma_{\gamma \in\langle P\rangle} \mathfrak{L}_{\gamma}\right)$, and let $\mathfrak{Z}^{P}$ be the centralizer of $\mathcal{L}^{P}$ in $\mathcal{L}_{0}$. in $\bigodot_{0}$.

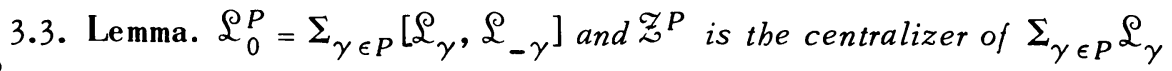

Proof. Suppose $Z \in \mathscr{L}_{0}$ centralizes $\Sigma_{\gamma \in P} \mathscr{\complement}_{\gamma}$ and suppose $\delta=\Sigma_{\gamma \in P} n_{\gamma} \gamma \epsilon$ $\langle P\rangle$. We must show that $\left[\mathfrak{Q}_{\delta}, \mathfrak{L}_{-\delta}\right] \subseteq \Sigma_{\gamma \in P}\left[\mathscr{\complement}_{\gamma}, \mathfrak{L}_{-\gamma}\right]$ and that $Z$ centralizes $\mathfrak{L}_{\delta}$. If $\Sigma_{n_{\gamma}}>0$, these assertions follow easily by induction on $\Sigma_{n_{\gamma}}$ (using 1.4). Suppose $\Sigma_{n_{\gamma}}<0$. Since $\left[\varrho_{\delta}, \mathfrak{L}_{-\delta}\right]=\left[\mathfrak{Q}_{-\delta}, \mathfrak{Q}_{\delta}\right]$, we need only show that $Z$ centralizes $\mathscr{L}_{\delta}$. But $Z$ centralizes $\mathcal{L}_{-\delta}$ and hence for $L_{\delta} \in \mathscr{L}_{\delta}$ and $L_{-\delta} \in \mathscr{L}_{-\delta}$, ([L $L_{\delta}$, $\left.Z], L_{-\delta}\right)=\left(L_{\delta},\left[Z, L_{-\delta}\right]\right)=0$. Thus, $\left[L_{\delta}, Z\right]=0$. of $\mathscr{L}_{0}$.

3.4. Proposition. (i) $\mathscr{L}_{0}=\mathscr{Z}^{P} \oplus \mathfrak{L}_{0}^{P}$, where $\mathscr{Z}^{P}$ and $\mathfrak{L}_{0}^{P}$ are orthogonal ideals

(ii) $\mathfrak{L}^{P}$ is a semisimple subalgebra of $\mathfrak{Q}$ with maximal split toral subalgebra $\mathcal{T} \cap \mathfrak{L}_{0}^{P}$ and $\mathfrak{T}=\left(\mathfrak{T} \cap \mathfrak{Z}^{P}\right) \oplus\left(\mathfrak{T} \cap \mathfrak{L}_{0}^{P}\right)$.

(iii) $\langle P\rangle$ is the root system of $\mathcal{L}^{P}$ relative to $\mathcal{T} \cap \mathfrak{L}_{0}^{P}$ and $\mathfrak{L}^{P}=\mathfrak{L}_{0}^{P} \oplus$ $\left(\Sigma_{\gamma \in\langle P\rangle} \mathfrak{Q}_{\gamma}\right)$ is the root decomposition.

Proof. (i) $\mathscr{Z}^{P}$ and $\mathscr{L}_{0}^{P}$ are certainly ideals of $\mathscr{L}_{0}$. Let $\left(\mathscr{Q}_{0}^{P}\right)^{\perp}$ be the set of all $Z \in \mathscr{L}_{0}$ which are orthogonal to $\mathscr{L}_{0}^{P}$. We must show that $\mathcal{E}^{P}=\left(\mathscr{Q}_{0}^{P}\right)^{\perp}$ and $\mathcal{Z}^{P} \cap \mathfrak{L}_{0}^{P}=(0)$. The first fact follows easily from the invariance of the Killing form. Suppose $Z \in \mathcal{J P}^{P} \cap \mathscr{Q}_{0}$. Then, $Z=\Sigma_{i}\left[M_{i}, N_{i}\right]$, where for each $i$ there exists $\gamma \in\langle P\rangle$ such that $M_{i} \in \mathfrak{L}_{\gamma}$ and $N_{i} \in \mathfrak{L}_{-\gamma}$. Since $Z \in \mathscr{Z}^{P}$, we have $\left[Z, M_{i}\right]=0$

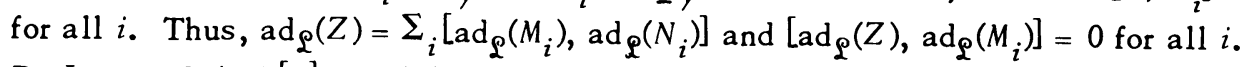
By Lemma 2.4 of $[1], \operatorname{ad}_{\varrho}(Z)$ is nilpotent and hence, by $1.11, Z=0$.

(ii) $\mathfrak{L}^{P}$ is certainly. a subalgebra of $\mathscr{L}$. To show that $\mathfrak{L}^{P}$ is semisimple, we show that any abelian ideal $R$ of $\mathcal{Q}^{P}$ is (0). By (i), $R$ is normalized by $\mathscr{L}_{0}$ and hence by $\mathcal{T}$. Thus, $R=\left(R \cap \mathscr{L}_{0}^{P}\right) \oplus\left(\Sigma_{\gamma \in\langle P\rangle} \mathfrak{R} \cap \mathfrak{L}_{\gamma}\right)$. Suppose for contradiction that $R \cap \mathfrak{L}_{\gamma} \neq(0)$ for some $\gamma \in\langle P\rangle$. Choose $X_{\gamma} \neq 0$ in $R \cap \mathfrak{L}_{\gamma}$. By 1.9, there exists $Y_{\gamma} \in \mathscr{L}_{-\gamma}$ such that $\left[X_{\gamma}, Y_{\gamma}\right]=T_{\gamma}$. Then, $Y_{\gamma}=1 / 2\left[\left[X_{\gamma}, Y_{\gamma}\right], Y_{\gamma}\right] \in \mathcal{R}$. But $\left[X_{y}, Y_{\gamma}\right] \neq 0$ giving a contradiction. Therefore, $\mathbb{R} \cap \mathscr{L}_{\gamma}=(0)$ for all $\gamma \in\langle P\rangle$. Thus, $\mathscr{R} \subseteq \mathscr{Q}_{0}$. But for $\gamma \in\langle P\rangle,\left[\mathscr{L}_{\gamma}, \mathfrak{R}\right] \subseteq \Re \cap \mathscr{L}_{\gamma}=(0)$ and hence $\mathscr{R} \subseteq \mathscr{Z}^{P}$. Thus, $R \subseteq \mathcal{Q}_{0} \cap \cap \mathscr{Z}^{P}=(0)$ and hence $\mathcal{R}=(0)$.

To prove that $\mathcal{T}=\left(\mathcal{T} \cap \mathscr{Z}^{P}\right) \oplus\left(\mathfrak{T} \cap \mathfrak{Q}_{0}^{P}\right)$, it suffices to show that if $T \in \mathcal{T}$, $T_{1} \in \mathscr{Z}^{P}, T_{2} \in \mathscr{L}_{0}$, and $T=T_{1}+T_{2}$, then $T_{2} \in \mathcal{T}$. Since $T_{2} \in \mathscr{L}_{0}$, it suffices to

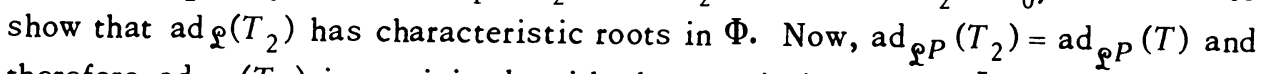
therefore ad $\operatorname{\varrho P}\left(T_{2}\right)$ is semisimple with characteristic roots in $\Phi$. Thus, we may 
imbed $T_{2}$ in a maximal split toral subalgebra of $\mathscr{\complement}^{\digamma}$. Thus, by $1.11, \operatorname{ad}_{\mathfrak{Q}}\left(T_{2}\right)$ has characteristic roots in $\Phi$.

$\mathcal{T} \cap \mathscr{L}_{0}^{P}$ is certainly a split toral subalgebra of $\mathfrak{L}^{P}$. Suppose $R$ is a split toral subalgebra of $\mathscr{L}^{P}$ containing $\mathcal{T} \cap \mathscr{L}_{0}^{P}$. Then, $\left(\mathcal{T} \cap \mathcal{J}^{P}\right) \oplus \mathscr{R}$ is a split toral subalgebra of $\mathcal{L}$ containing $\mathcal{T}$ and hence $\left(\mathcal{T} \cap \mathcal{Z}^{P}\right) \oplus \mathscr{R}=\mathfrak{T}$. Thus, $\mathcal{T} \cap \mathscr{L}_{0}^{P}=\mathfrak{R}$.

(iii) Since $\mathfrak{T}=\left(\mathcal{T} \cap \mathscr{Z}^{P}\right) \oplus\left(\mathcal{T} \cap \mathcal{L}_{0}^{P}\right)$, we may identify each element of $\langle P\rangle$ with its restriction to $\mathcal{J} \cap \mathscr{L}_{0}^{P}$. (Each such element is zero on $\mathcal{T} \cap \mathscr{Z}^{P}$.) (iii) is then clear.

3.5. By 2.2 , we have $\mathfrak{L}_{0}^{P}=\left(\complement_{0}^{P} \cap \mathcal{C}\right) \oplus\left(\mathfrak{Q}_{0}^{P} \cap\left[\complement_{0}, \mathfrak{L}_{0}\right]\right)$. We define

$$
\mathbb{Q P}^{P}=\left[\mathscr{L}_{0}^{P}, \mathfrak{L}_{n}^{P}\right]=\mathfrak{L}_{0}^{P} \cap\left[\mathscr{L}_{0}, \mathfrak{L}_{0}\right]
$$

in which case $\mathfrak{Q}^{P}$ is the anisotropic kernel of $\mathscr{L}^{P}$ and $\mathscr{L}_{0}^{P}=\left(\mathscr{Q}_{0}^{P} \cap \mathcal{C}\right) \oplus \mathbb{Q}^{P}$.

3.6. Let $\gamma \in \Sigma$. Applying the definitions of 3.2 and 3.5 to $P=\{\gamma\}$, we define $\mathfrak{Q}^{\gamma}, \mathfrak{Q}_{0}^{\gamma}, \mathfrak{Z}^{\gamma}$, and $\mathfrak{Q} \gamma$. If $\gamma, \delta, \gamma+\delta \in \Sigma$, since $\mathfrak{L}_{\gamma+\delta}=\left[\mathfrak{Q}_{\gamma}, \mathfrak{L}_{\delta}\right]$, we have $\mathfrak{Z}^{\gamma} \cap$ $Z^{\delta} \subseteq \mathcal{Z}^{+}{ }^{+\delta}$.

3.7. Lemma. Let $y \in \Sigma$ and let $\mathfrak{Q}$ be a nonzero ideal of $\mathfrak{Q}_{0}^{\gamma}$. Then, $\mathfrak{Q}$ does not commute with any nonzero element of $\mathfrak{L}_{\gamma}$.

Proof. Let $\mathbb{M}=\left\{L_{\gamma} \in \mathfrak{L}_{\gamma}:\left[L_{\gamma}, \mathbb{Q}\right]=(0)\right\}$. We must show that $\mathbb{M}=(0)$. Since

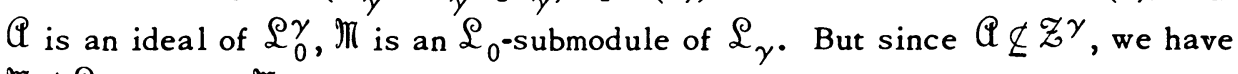
$\mathbb{M} \neq \mathscr{L}_{\gamma}$. Thus, $\mathbb{M}=(0)$.

3.8. By 3.3, we have $\mathfrak{L}_{0}^{P}=\Sigma_{\gamma \in P^{2}} \mathscr{Q}_{0}^{\gamma}$ and hence $\mathfrak{A}^{P}=\Sigma_{\gamma \in P} \mathfrak{A}^{\gamma}$. But $\mathfrak{L}^{\Pi}$ is certainly an nonzero ideal of $\mathscr{L}$ and hence $\mathscr{L}=\mathfrak{L}^{\Pi}$. Thus, $\mathfrak{L}_{0}=\Sigma \gamma \in \mathbf{\Pi}^{\mathfrak{L}}{ }_{0}$ and $\left[\mathfrak{Q}_{0}, \mathfrak{L}_{0}\right]=\Sigma_{\gamma \in \mathbf{n}} \mathfrak{Q}^{\gamma}$.

4. The centrum of $\varrho$. The centralizer $\Gamma$ of $\operatorname{ad}_{\varrho}(£)$ in $\operatorname{End}_{\Phi}(£)$ is called the centrum of $\mathscr{L}$. (See Chapter $X$ of [1].) $\Gamma$ is a field and $\mathcal{L}$ is an algebra over $\Gamma$, where scalar multiplication by $c \in \Gamma$ is defined by $c L=L^{c}$ for $L \in \mathscr{Q}$. $\mathcal{Q}$ is said to be central if $\Gamma=\Phi$.

4.1. Proposition. (i) $\Gamma \mathcal{T}$ is a maximal split toral subalgebra of $\mathfrak{L} / \Gamma$.

(ii) The map $\Gamma \otimes_{\Phi} \mathcal{T} \rightarrow \Gamma \mathcal{T}$ is a $\Phi$-isomorphism (and bence we may identify $\Phi$-linear maps $\mathfrak{T} \rightarrow \Phi$ with their extensions to $\Gamma$-linear maps $\Gamma \mathcal{T} \rightarrow \Gamma$ ).

(iii) With the identification of (ii), $\mathcal{T}$ and $\Gamma \mathcal{T}$ bave the same root systems and root decompositions.

(iv) If $\mathfrak{A}$ is an ideal of $\left[\mathscr{Q}_{0}, \mathfrak{L}_{0}\right] / \Phi$, then $\mathbb{Q}$ is an ideal of $\left[\mathfrak{Q}_{0}, \mathfrak{L}_{0}\right] / \Gamma$.

Proof. (i) $\Gamma \mathcal{J}$ is clearly a split toral subalgebra of $\mathcal{L} / \Gamma$. Suppose $R$ is a split toral subalgebra of $\mathscr{L} / \Gamma$ containing $\Gamma \mathcal{J}$. By $1.10, \mathscr{R}$ has a $\Gamma$-basis consisting of elements $R$ such that $\operatorname{ad}_{\mathfrak{l}}(R)$ has integral characteristic roots. Each of these elements must lie in $\mathcal{T}$ and hence $R=\Gamma \mathcal{T}$.

(ii) and (iii) Let $\gamma \in \Sigma \cup\{0\}$. Then, for $c \in \Gamma, T \in \mathcal{T}$, and $L_{\gamma} \in \mathfrak{L}_{\gamma}$, we have 
$\left[L_{\gamma}, c T\right]=c\left[L_{\gamma}, T\right]=c \gamma(T) L_{\gamma}$. Thus, there exists a $\Gamma$-linear map $\Gamma \mathcal{J} \rightarrow \Gamma$, which we temporarily denote by $\tilde{\gamma}$, such that $\mathscr{L}_{\gamma} \subseteq \mathcal{L}_{\tilde{\gamma}}$ and $\tilde{\gamma}(c T)=c \gamma(T)$ for $c \in \Gamma$ and $T \in \mathcal{T}$. Thus, $\mathscr{L}=\Sigma \mathscr{Q} \tilde{\gamma}$ and, since the $\tilde{\gamma}$ are evidently distinct, this sum is direct. But $\mathfrak{L}=\bigoplus \Sigma \mathscr{L}_{\gamma}$ and hence $\mathscr{L}_{\gamma}=\mathfrak{L}_{\tilde{\gamma}}$ for $\gamma \in \Sigma \cup\{0\}$. Hence, $T_{\gamma}=T \tilde{\gamma}$ for $\gamma \in \Sigma$. Therefore,

$$
\begin{aligned}
\operatorname{dim}_{\Gamma}\left(\Gamma^{\mathcal{S}}\right) & =\operatorname{dim}_{Q}\{Q \text {-space generated by } T \tilde{\gamma}, \gamma \in \Sigma\} \\
& =\operatorname{dim}_{Q}\left\{Q \text {-space generated by } T_{\gamma}, \gamma \in \Sigma\right\}=\operatorname{dim}_{\Phi}(\mathcal{T}) .
\end{aligned}
$$

(ii) and (iii) are then immediate.

(iv) $\mathfrak{A}=\left[\mathfrak{A}, \mathfrak{L}_{0}\right]$ and therefore $\Gamma \mathfrak{A}=\Gamma\left[\mathfrak{A}, \mathfrak{L}_{0}\right]=\left[\mathfrak{Q}, \Gamma \mathfrak{L}_{0}\right]=\left[\mathfrak{Q}, \mathfrak{L}_{0}\right]=\mathfrak{Q}$.

4.2. Proposition. Let $\gamma \in \Sigma$ and let $\Gamma_{\gamma}$ be the centralizer of $\varrho_{0}^{\rho} \gamma$ in $\operatorname{End}_{\boldsymbol{\phi}}\left(\varrho_{\gamma}\right)$. Then, the restriction map defines a monomorpbism $\Gamma \rightarrow \Gamma_{\gamma}$ of $\Phi$-algebras. If $\gamma$ is conjugate under $W$ to the bighest root of $\Sigma$, then this map is an isomorphism, $\Gamma T_{\gamma}=$ $\mathcal{C} \cap \mathfrak{L}_{0}^{\gamma}$, and $\mathfrak{L}_{0}^{\gamma}=\Gamma T_{\gamma} \oplus \mathbb{Q}^{\gamma}$.

Proof. Since elements of $\Gamma$ commute with $\operatorname{ad}_{\mathcal{\rho}}(\mathcal{T})$, the above restriction map makes sense, i.e. the root spaces of $\mathcal{L}$ are stabilized by elements of $\Gamma$. Since $\Gamma$ is a field the map is a monomorphism of $\Phi$-algebras. Denote the image of an element $c$ of $\Gamma$ in $\Gamma_{\gamma}$ by $\tilde{c}$ and denote the image of $\Gamma$ in $\Gamma_{\gamma}$ by $\tilde{\Gamma}$. Suppose now that $\gamma$ is conjugate under $\bar{C}$ to the highest root of $\Sigma$. By rechoosing $\Pi$, we may assume $\gamma$ is the highest root of $\Sigma$. Since $\tilde{\Gamma} \subseteq \Gamma_{\gamma}$, the centralizer of $\Gamma_{\gamma}$ in $\operatorname{End}_{\Phi}\left(\varrho_{\gamma}\right)$ is contained in the centralizer of $\tilde{\Gamma}$. To show that $\tilde{\Gamma}=\Gamma_{\gamma}$, it suffices to show that these two centralizers are equal. Suppose $\theta \in \operatorname{End}_{\Phi}\left(\mathfrak{L}_{\gamma}\right)$ centralizes $\widetilde{\Gamma}$. We may extend $\theta$ to a $\Phi$-linear map $\mathscr{L} \stackrel{\psi}{\rightarrow} \mathscr{L}$ which centralizes $\Gamma$, by putting $\theta$ equal to zero on $\mathscr{L}_{0}$ and on all root spaces of $\mathscr{L}$ other than $\mathscr{L}_{\gamma}$. Let $\mathscr{\mathcal { P }} \stackrel{\rho}{\rightarrow} \mathrm{End}_{\Phi}(\mathcal{L})$ be the adjoint representation of $\mathcal{Q}$. For any Lie algebra $\mathbb{Q}$, let $u(\mathbb{Q})$ denote the universal enveloping algebra of $\mathscr{Q}$. Then, $\rho$ (resp. $\rho_{\gamma}$ ) extends to a representation $u(\mathcal{L}) \stackrel{\rho}{\longrightarrow}$

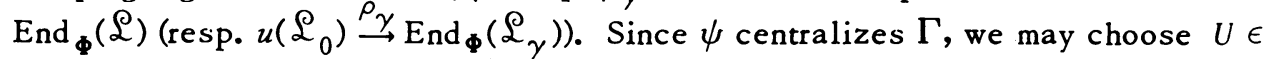
$u(\mathfrak{Q})$ such that $U^{\rho}=\psi$. Let $\mathcal{Q}^{+}$(resp. $\mathcal{Q}^{-}$) be the sum of the positive (resp. negative) root spaces of $\mathcal{L}$. Then, by the Poincaré-Birkhoff-Witt theorem we may write

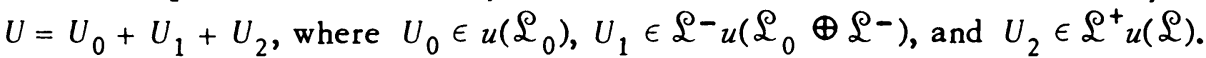
But $U_{0}^{\rho}$ stabilizes $\mathscr{L}_{\gamma}, U_{1}^{\rho}$ maps $\mathscr{L}_{\gamma}$ into $\mathscr{L}_{0}+\Sigma_{\delta \epsilon \Sigma-\{\gamma\}} \mathscr{L}_{\gamma}$, and $U_{2}^{\rho}$ is zero on $\mathfrak{Q}_{\gamma}$. Thus, $\theta=\psi\left|\complement_{\gamma}=U^{\rho}\right| \aleph_{\gamma}=U_{0}^{\rho}\left|\mathcal{\complement}_{\gamma}=U_{0}^{\rho_{\gamma}}\right| \aleph_{\gamma}$. Therefore, $\theta \in u\left(\aleph_{0}\right)^{\rho_{\gamma}}$ and hence $\theta$ centralizes $\Gamma_{\gamma}$ as required.

It is easy to check that $\left(c T_{\gamma} / 2\right)^{\rho} \gamma=\tilde{c}$ for $c \in \Gamma$. Thus, $\tilde{\Gamma}=\left(\Gamma T_{\gamma}\right)^{\rho} \underline{C}$ $\left(\Gamma\left(\mathcal{Q} \cap \mathfrak{L}_{0}^{\gamma}\right)\right)^{\rho_{\gamma}}=\left(\mathcal{C} \cap \mathfrak{L}_{0}^{\gamma}\right)^{\rho_{\gamma}} \subseteq \Gamma_{\gamma}$ and since $\tilde{\Gamma}=\Gamma_{\gamma}$ and $\rho_{\gamma}$ is faithful on $\overline{\mathfrak{L}}_{0}^{\gamma}$, we have $\Gamma T_{\gamma}=\mathcal{C} \cap \mathfrak{Q}_{0}^{\gamma}$. But by 3.5 , we have $\mathfrak{L}_{0}^{\gamma}=\left(\mathcal{C} \cap \mathfrak{L}_{0}^{\gamma}\right) \oplus \mathfrak{A}^{\gamma}=\Gamma T_{\gamma} \oplus \mathfrak{A}^{\gamma}$. Since $\mathcal{L}_{0}^{\rho_{\gamma}}=\left(\varrho_{0}^{\gamma \gamma}\right)^{\rho_{\gamma}}$ we have the following:

4.3. Corollary. Suppose $\gamma \in \Sigma$ and $\gamma$ is conjugate under 60 to the bighest root of $\Sigma$. Then, the following are equivalent: 
(i) $\mathcal{L}$ is central,

(ii) $\mathfrak{Q}^{\gamma}$ is central,

(iii) $\mathscr{L}_{0}^{\rho_{\gamma}}$ generates $\mathrm{End}_{\Phi}\left(\mathcal{Q}_{\gamma}\right)$ as an associative algebra with 1.

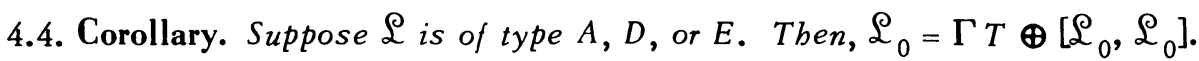

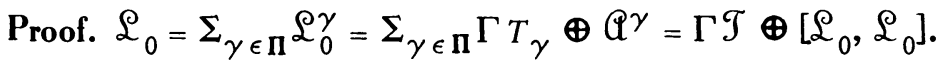

5. The automorphism $\phi(w)$. In this section, we recall the definition of certain automorphisms of $\mathcal{L}$ corresponding to elements of $\mathcal{W}$ and record some facts about these automorphisms. (See for example p. 38 of [4].)

5.1. Proposition. Let $w \in W$. Then, there exists $\phi(w) \in G$ such that $\xi^{2}\left(T^{\phi(w)}\right)=\xi(T)$ for $T \in \mathcal{T}$ and $\xi \in \chi_{\Sigma}$. Any two sucb automorphisms differ by an automorphism which is inner on $\mathfrak{Q}_{0}$.

Proof. The last statement follows from 2.5. It clearly suffices to prove the first statement for $w^{2}=w_{\gamma}$, where $\gamma \in \Sigma$. Choose $X_{\gamma} \neq 0$ in $\mathfrak{L}_{\gamma}$ and $Y_{\gamma} \neq 0$ in $\stackrel{\complement}{-\gamma}_{\text {such that }}\left[X_{\gamma}, Y_{\gamma}\right]=T_{\gamma}$. Then,

$$
\phi\left(\omega_{\gamma}\right)=\exp \left(\operatorname{ad}\left(Y_{\gamma}\right)\right) \exp \left(\operatorname{ad}\left(X_{\gamma}\right)\right) \exp \left(\operatorname{ad}\left(Y_{\gamma}\right)\right)
$$

is the required automorphism (p. 39 of [4]).

5.2. It follows from the proof of 5.1 that for each $\gamma \in \Sigma$, we may choose $\phi\left(w_{\gamma}\right)$ in such a way that the elements of $\mathcal{Z}^{\gamma}$ are fixed by $\phi\left(w_{\gamma}\right)$.

5.3. We assume from now on that for each $w \in W$ there is some fixed choice of $\phi(\omega)$ satisfying 5.1. The following is clear:

5.4. Proposition. Let $w \in \mathcal{W}$. Then, $\mathfrak{L}_{0}^{\phi(w)}=\mathfrak{L}_{0}$ and $\mathfrak{L}_{\gamma}^{\phi(w)}=\mathcal{L}_{\gamma^{w}}$ for $\gamma \in \Sigma$.

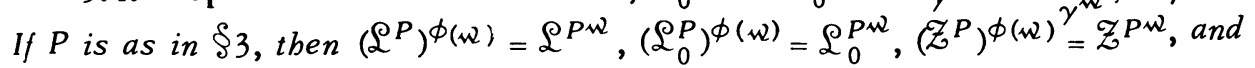
$\left(\mathfrak{Q}^{P}\right)^{\phi(\mathcal{N})}=\mathfrak{Q}^{P^{\mathcal{W}}}$.

5.5. Lemma. Suppose $\gamma \in \Sigma$ and $2 \gamma \notin \Sigma$. Then, $\left(\mathcal{Q}_{0}^{\gamma} \cap \mathcal{C}\right)=\left\{L_{0} \in \mathcal{C}: L_{0}^{\phi\left(\omega^{2} \gamma\right)}=\right.$ $\left.-L_{0}\right\}$ and $\left(\mathcal{Z}^{\gamma} \cap \mathcal{C}\right)=\left\{L_{0} \in \mathcal{C}: L_{0}^{\phi(w \gamma)}=L_{0}\right\}$.

Proof. By 5.1, the conclusion of the lemma does not depend on our choice of $\phi\left(w_{\gamma}\right)$. Hence, we may assume $\phi\left(w_{\gamma}\right)$ fixes the elements of $\mathcal{E} \gamma$. It remains to show that $L_{0}^{\phi\left(w^{2} \gamma\right)}=-L_{0}$ for $L_{0} \in \mathscr{L}_{0}^{\gamma} \cap \mathcal{C}$. But, by $4.2, \mathcal{L}_{0}^{\gamma} \cap \mathcal{C}=\Gamma^{\gamma} T_{\gamma}$, where $\Gamma^{\gamma}$ is the centrum of $\mathscr{L}^{\gamma}$. This clearly implies what we are after.

5.6. We assume for the moment that $\mathcal{Q}$ has rank $1, \mathcal{L}$ is reduced, $\Pi=\{\gamma\}$, and

$$
\phi=\exp (\operatorname{ad}(Y)) \exp (\operatorname{ad}(X)) \exp (\operatorname{ad}(Y)),
$$

where $X \neq 0$ in $\mathfrak{E}_{\gamma}, Y \neq 0$ in $\mathfrak{L}_{-\gamma}$, and $[X, Y]=T_{\gamma}$. Write $\mathcal{S}=\Phi X \oplus \Phi T_{\gamma} \oplus \Phi Y$. By the representation theory for $\mathcal{S}$, we may choose a basis for $\mathcal{L}$ as follows: 


$$
\begin{aligned}
& X_{0} X_{1} \cdots X_{n} \\
& S_{1} S_{2} \cdots S_{m} \quad A_{0} A_{1} \cdots A_{n} \\
& \\
& Y_{0} Y_{1} \cdots Y_{n},
\end{aligned}
$$

where the vertical lines are each bases for $\delta_{\text {-irreducibles; }} A_{i}=\left[X_{i}, Y\right], Y_{i}=\left[A_{i}\right.$, $Y](i=0, \cdots, n)$; and the horizontal lines are bases for (from top to bottom) $\mathscr{L}_{\gamma}$, $\mathscr{L}_{0}$ and $\mathscr{L}_{-\gamma}$. We note that each $S_{i}$ commutes with $X$ and $Y$ and hence $S_{i}^{\phi}=S_{i}$, $i=1, \cdots, m$. If $\pi_{i}=\Phi X_{i} \oplus \Phi A_{i} \oplus \Phi Y_{i}$, one easily checks that the matrix of $\phi \mid \Re_{i}$ relative to the basis $X_{i}, A_{i}, Y_{i}$ is

$$
\left(\begin{array}{rrr}
0 & 0 & 1 / 2 \\
0 & -1 & 0 \\
2 & 0 & 0
\end{array}\right)
$$

$i=0, \cdots, n$. Thus, $\phi^{2}=1, A_{i}^{\phi}=-A_{i}(i=0, \cdots, n)$, and $\phi$ is diagonalizable with characteristic roots 1 and -1 .

Suppose now that $I$ is a subalgebra of $\mathscr{Q}_{0}$ stabilized by $\phi$. Then, we may write $I_{=} I_{+} \oplus I_{-}$, where $g_{+}$(resp. $g_{-}$) is the eigenspace for $\phi$ corresponding to the eigenvalue 1 (resp. -1 ). From the previous paragraph it follows that

$$
I_{+}=\left\{L_{0} \in g_{:}\left[L_{0}, X\right]=0\right\}=\left\{L_{0} \in g_{:}\left[L_{0}, Y\right]=0\right\} .
$$

One also checks easily that $\mathscr{I}_{+}$and $\mathscr{I}_{-}$are orthogonal to each other. We have

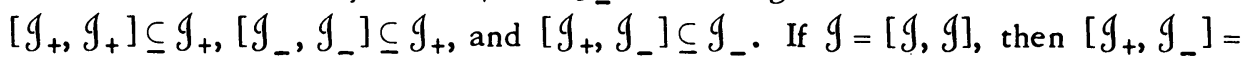
I.

Finally, we note that $A_{0}, \cdots, A_{n}$ is a basis for $\left(\complement_{0}\right)_{-}$and $X_{0}, \cdots, X_{n}$ is a basis for $£_{\gamma}$, and therefore $\operatorname{dim}_{\Phi}\left(\varrho_{\gamma}\right)=\operatorname{dim}_{\Phi}\left(\left(\varrho_{0}\right)_{-}\right)$.

6. Reduced, rank 1 algebras and their applications.

6.1. Theorem. Suppose $\mathcal{Q}$ is a reduced rank 1 simple Lie algebra and $\Pi=\{y\}$. Then, the only ideals of $\left[\mathfrak{C}_{0}, \mathfrak{L}_{0}\right]$ stabilized by $\phi\left(\omega_{\gamma}\right)$ are $(0)$ and $\left[\mathfrak{\complement}_{0}, \mathfrak{L}_{0}\right]$.

Proof. By 4.1, we may assume $\mathcal{Q}$ is central and hence, by $4.4, \mathfrak{L}_{0}=\mathcal{T} \oplus\left[\mathfrak{Q}_{0}\right.$, $\left.\mathcal{L}_{0}\right]$. We use the notation of 5.6 and we may assume $\phi\left(w_{\gamma}\right)=\phi$. Suppose for contradiction that there exists a proper nonzero ideal $\mathscr{Q}$ of $\left[\mathscr{Q}_{0}, \mathfrak{Q}_{0}\right]$ which is stabilized by $\phi$. There is an ideal $\mathfrak{B}$ of $\left[\complement_{0}, \mathfrak{L}_{0}\right]$ such that $\mathscr{A} \oplus \mathfrak{B}=\left[\mathscr{\complement}_{0}, \mathfrak{L}_{0}\right]$. Then, $\mathfrak{B}^{\phi}=\mathfrak{B}$ and

$$
\mathfrak{L}_{0}=\mathfrak{A}_{+} \oplus \mathfrak{B}_{+} \oplus \mathfrak{T} \oplus \mathbb{A}_{-} \oplus \mathfrak{B}_{-} \text {, }
$$

where the summands are mutually orthogonal subspaces of $\mathscr{L}_{0}$.

For any $\Phi$-subspace $\mathcal{I}$ of $\mathscr{L}_{0}$, put $\mathcal{G}=[\mathscr{I}, X]$ and $\hat{\mathscr{I}}=[\mathcal{G}, Y]$. By 5.5, we 
have $\mathfrak{L}_{\gamma}=\Phi X+\hat{\mathfrak{Q}}_{-}+\hat{\mathscr{B}}_{-}$and $\mathfrak{\mathscr { L }}_{-\gamma}=\Phi Y+\check{\mathscr{Q}}_{-}+\check{\mathscr{B}}_{-}$. We note now that it suffices to show $\left[\hat{\mathbb{Q}}_{-}, \mathfrak{B}_{-}\right]=(0)$. For one easily checks that $\left[\hat{\mathbb{Q}}, \mathscr{B}_{+}\right]=(0)$ and hence $\left[\hat{\mathbb{Q}}_{-}, \mathfrak{B}\right]=(0)$. But then by 3.7 , we have $\hat{\mathbb{Q}}_{-}=(0)$ and hence, $\left[\mathfrak{Q}_{-}, X\right]=(0)$. Therefore, since $\left[\mathfrak{Q}_{+}, X\right]=(0)$, we have $[\mathscr{Q}, X]=(0)$ and hence, by $3.7, \mathscr{Q}=(0)$ (a contradiction). Thus, we must show that $\left[\hat{\mathscr{A}}_{-}, \mathfrak{B}_{-}\right]$is orthogonal to $Y, \check{\mathbb{Q}}_{-}$, and 乃

For $\hat{A}_{-} \epsilon \hat{\mathfrak{Q}}_{-}, \check{A}_{-} \epsilon \check{\mathfrak{Q}}_{-}, B_{-} \in \mathscr{B}_{-}$, and $B_{+} \in \mathscr{B}_{+}$, we have $\left(\left[\hat{A}_{-}, \check{A}_{-}\right],\left[B_{-}\right.\right.$, $\left.\left.B_{+}\right]\right)=\left(\left[\hat{A}_{-}, B_{-}\right],\left[\check{A}_{-}, B_{+}\right]\right)+\left(\left[\bar{A}_{-}, B_{-}\right],\left[B_{+}, \hat{A}_{-}\right]\right)=0$, since $\left[\check{\mathscr{C}}_{-}, \mathscr{B}_{+}\right]=(0)$ and $\left[\hat{\mathscr{Q}}, \mathfrak{B}_{+}\right]=(0)$. Thus, since $\mathscr{B}_{-}=\left[\mathfrak{B}_{-}, \mathfrak{B}_{+}\right]$, we have $\left[\hat{\mathscr{A}}_{-}, \check{\mathscr{A}}_{-}\right]$orthogonal to $\mathfrak{B}_{-}$. Similarly, $\left[\hat{\mathscr{B}}_{-}, \check{\mathscr{B}}_{-}\right]$is orthogonal to $\mathscr{Q}_{-}^{-}$. From the first of these facts, it follows that $\breve{\mathscr{A}}_{-}$is orthogonal to $\left[\hat{\mathscr{A}}_{-}, \mathscr{B}_{-}\right]$and from the second, it follows that $\breve{\mathscr{B}}_{-}$is orthogonal to $\left[\mathfrak{Q}_{-}, \hat{\mathfrak{B}}_{-}\right]=\left[\hat{\mathscr{A}}_{-}^{-}, \hat{B}_{-}^{-}\right]$. It remains to show that $\left[\hat{\mathscr{A}}_{-}, \mathfrak{B}_{-}\right]$is orthogonal to $Y$. This however follows from the fact that $\mathbb{A}_{-}$and $\mathfrak{B}_{-}$are orthogonal.

6.2. Corollary. Suppose $\mathcal{Q}$ is a reduced rank 1 simple algebra with $\Pi=\{y\}$ and suppose $\mathfrak{A}$ is a proper nonzero ideal of $\left[\mathfrak{\complement}_{0}, \mathfrak{L}_{0}\right]$. Then, $\left[\mathfrak{\complement}_{0}, \mathfrak{L}_{0}\right]=\mathbb{Q} \oplus$ $\mathbb{Q}^{\phi\left(\aleph_{\gamma}\right)}$ and $\operatorname{dim}_{\Gamma}(\mathfrak{Q})=\operatorname{dim}_{\Gamma}\left(\mathcal{L}_{\gamma}\right)-1$.

Proof. We may assume $\phi\left(w_{\gamma}\right)=\phi$ (as in 5.6). Since $\phi^{2}=1, \mathfrak{Q}+\mathfrak{Q}^{\phi}$ and $\mathfrak{Q} \cap$ $\mathbb{Q}^{\phi}$ are stabilized by $\phi$. Thus, $\mathfrak{Q}+\mathfrak{Q}^{\phi}=\left[\mathfrak{Q}_{0}, \mathfrak{L}_{0}\right]$ and $\mathfrak{Q} \cap \mathfrak{Q}^{\phi}=(0)$. Hence, $\left[\mathfrak{Q}_{0}, \mathfrak{L}_{0}\right]_{=} \mathbb{Q} \oplus \mathbb{Q}^{\phi}$. Now, $\left[\mathfrak{L}_{0}, \mathfrak{L}_{0}\right]_{-}=\left\{A-A^{\phi}: A \in \mathbb{A}\right\}$ and hence $\operatorname{dim}_{\Gamma}\left(\mathfrak{Q}_{\gamma}\right)=$ $\operatorname{dim}_{\Gamma}\left(\left(\mathscr{\complement}_{0}\right)_{-}\right)=\operatorname{dim}_{\Gamma}\left(\left[\mathscr{L}_{0}, \mathfrak{L}_{0}\right]_{-}\right)+1=\operatorname{dim}_{\Gamma}(\mathscr{Q})+1$.

We now drop the assumption that $\mathcal{L}$ has rank 1 and prove

6.3. Lemma. Let $\mathbb{A}$ and $\mathfrak{B}$ be simple ideals of $\left[\mathfrak{\complement}_{0}, \mathfrak{L}_{0}\right]$. Then, there exists $\gamma \in \Sigma$ sucb that $\mathbb{A}+\mathfrak{B} \subseteq \mathbb{Q}^{\gamma}$.

Proof. We may assume $\mathfrak{Q} \neq \mathfrak{B}$. We induct on the rank $r$ of $\mathscr{L}$. If $r=1$, the result is clear. Suppose $r>1$. We may choose $\gamma_{1}, \cdots, \gamma_{s} \in \Pi$ so that $\gamma_{i}+\gamma_{i+1} \in \Sigma$ for $i=1, \cdots, s-1, \mathfrak{Q} \subseteq \mathbb{Q}^{\gamma}$ and $\mathfrak{B} \subseteq \mathbb{Q}^{\gamma} s$. By the induction hypothesis we may assume $s=r$ and $\mathfrak{Q} \notin \mathbb{Q} \gamma_{2}+\ldots+\gamma_{r}$ Thus since $\mathfrak{Q}$ is simple, $\mathfrak{Q} \subseteq \mathfrak{Z}^{\gamma_{2}}+\cdots+\gamma_{r}$. But $\mathbb{Q} \not \mathbb{Z}^{\gamma_{1}}$ and $\mathcal{Z}^{\gamma_{1}} \supseteq \mathcal{Z}^{-\left(\gamma_{2}+\cdots+\gamma_{r}\right)} \cap \mathcal{Z}^{\gamma_{1}+\cdots+\gamma_{r}}=\mathcal{Z}^{\gamma_{2}}+\cdots+\gamma_{r}$ $\mathscr{Z}^{\gamma_{1}+\cdots+\gamma_{r}}$ and hence $\mathfrak{A} \notin \mathcal{Z}^{\gamma_{1}+\cdots+\gamma_{r}}$. Therefore, $\mathbb{A} \subseteq \mathbb{A}^{\gamma_{1}+\cdots+\gamma_{r}}$. Similarly, we may assume $\mathfrak{B} \subseteq \mathbb{A}^{\gamma_{1}+\cdots+\gamma_{r}}$. Put $\gamma=\gamma_{1}+\cdots+\gamma_{r}$.

6.4. Corollary. Suppose $\mathcal{Q}$ is a reduced simple Lie algebra. Then, the simple summands of $\left[\mathfrak{L}_{0}, \mathfrak{L}_{0}\right]$ are conjugate under $G$.

Proof. Let $\mathfrak{A}$ and $\mathfrak{B}$ be simple summands of $\left[\mathfrak{Q}_{0}, \mathfrak{L}_{0}\right]$. Choose $\gamma \in \Sigma$ such that $\mathfrak{A}+\mathfrak{B} \subseteq \mathbb{A}^{\gamma}$. By 6.2, we have either $\mathbb{A}=\mathfrak{B}$ or $\mathbb{A}^{\phi\left(\mathfrak{w}^{\gamma} \gamma\right)}=\mathfrak{B}$.

6.5. Corollary. Suppose $\mathcal{Q}$ is of type $D$ or E. Then $\left[\mathscr{Q}_{0}, \mathfrak{L}_{0}\right]=(0)$.

Proof. We may choose a subset $P=\left\{\gamma_{1}, \gamma_{2}, \gamma_{3}, \gamma_{4}\right\}$ of $\Pi$ with Dynkin diagram 


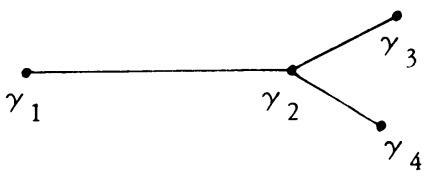

Suppose $\left[\mathfrak{L}_{0}, \mathfrak{L}_{0}\right] \neq(0)$. Then, since all roots of $\Sigma$ are conjugate under $\mathbb{W}$, we have $\mathbb{A}^{\gamma_{2}} \neq(0)$. Thus, we must have $\mathbb{A}^{\gamma} \cap \cap \mathbb{A}^{\gamma} \neq(0)$. (For if $\mathfrak{A}^{\gamma_{1}} \cap \mathbb{Q}^{\gamma_{2}}=(0)$, then $\mathbb{Q}^{\gamma_{1}} \subseteq \mathfrak{Z}^{\gamma_{2}}$ and therefore, by $5.2, \mathbb{Q}^{\gamma_{1}}=\left(\mathbb{Q}^{\gamma_{1}}\right)^{\phi\left(\mathfrak{w}^{2} \gamma_{2}\right)}=\mathbb{A}^{\gamma_{1}+\gamma_{2}}$. Similarly, $\mathbb{Q}^{\gamma_{2}}=\mathbb{Q}^{\gamma_{1}+\gamma_{2}}$ and hence $\mathbb{Q}^{\gamma_{2}}=\mathbb{Q}^{\gamma} \cap \cap \mathbb{Q}^{\gamma_{2}}=(0)$, a contradiction.) Similarly,

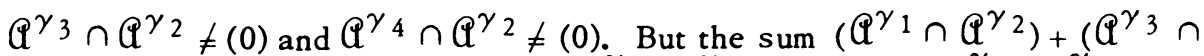
$\left.\mathfrak{Q}^{\gamma_{2}}\right)+\left(\mathfrak{Q}^{\gamma_{2}} \cap \mathfrak{Q}^{\gamma_{2}}\right)$ is direct. (Indeed $\mathfrak{Q}_{0}^{\gamma_{1}} \subseteq \mathfrak{Z}^{\gamma_{3}}$ and therefore $\mathfrak{Q}_{0}^{\gamma_{1}} \cap \mathfrak{Q}_{0}^{\gamma_{3}}=(0)$. Similarly, $\mathfrak{Q}_{0}^{\gamma} \cap \cap \mathfrak{L}_{0}^{\gamma / 4}=(0)$ and $\mathfrak{Q}_{0}^{\gamma} \cap \cap \mathfrak{Q}_{0}^{\gamma}{ }_{4}=(0)$.) Thus, $\mathfrak{Q}^{\gamma / 2}$ contains at least three nonzero distinct simple ideals, contradicting 6.2.

\subsection{Corollary. A central simple Lie algebra of type $D$ or $E$ is split.}

Proof. This is a consequence of 6.5 and 4.4.

7. Extension results.

7.1. If $V$ is an $M$-module and $V^{\prime}$ is an $\mathbb{M}^{\prime}$-module, where $M$ and $\mathbb{M}^{\prime}$ are Lie algebras, and if $M \stackrel{\theta}{\rightarrow} \mathbb{M}^{\prime}$ is a Lie isomorphism, then a $\theta$-equivalence of $V$ onto $V^{\prime}$ is a linear bijection $V \stackrel{\eta}{\rightarrow} V^{\prime}$ such that $(v M)^{\eta}=v^{\eta} M^{\theta}$ for $v \in V$ and $M \in \Re$.

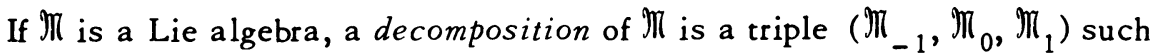
that $\mathbb{M}_{-1}, \mathbb{M}_{0}, \mathbb{M}_{1}$ are subalgebras of $\mathbb{M}_{,}\left[\mathbb{M}_{1}, M_{0}\right] \subseteq \mathbb{M}_{1}$, and $\left[\mathbb{M}_{-1}, M_{0}\right] \subseteq \mathbb{M}_{-1}$. If $V$ is an $\mathbb{M}$-module, we denote by $V^{N_{1}}$ the set of all $v \in V$ which are annihilated by $\pi_{1} . V^{\pi_{1}}$ is an $\pi_{0}$-submodule of $V . N$. R. Wallach (see [7] and [8]) has studied these decompositions and we use his results to prove:

7.2. Proposition. Suppose $\mathbb{M}$ and $\mathbb{M}^{\prime}$ are Lie algebras with decompositions

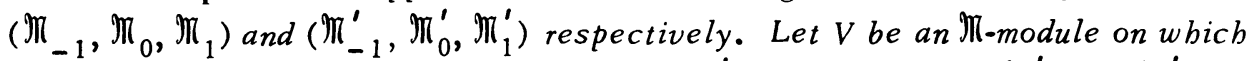
$M_{-1}^{-1}$ and $M_{1}$ act nilpotently and let $V^{\prime}$ be an $M^{\prime}$-module on which $M_{-1}^{\prime}$ and $M_{1}^{\prime}$ act nilpotently. Let $\mathbb{M} \stackrel{\theta}{\rightarrow} \mathbb{M}^{\prime}$ be an isomorphism which preserves the decompositions (i.e. $\mathbb{M}_{i}^{\theta}=\mathbb{M}_{i}^{\prime}$ for $\left.i=-1,0,1\right)$. Put $\theta_{0}=\theta \mid M_{0}$. Then, any $\theta_{0}$-equivalence $V^{M_{1} \rightarrow}$ $V^{\prime}$ m $_{1}$ extends to a $\theta$-equivalence $V \rightarrow V^{\prime}$.

Proof. We may assume without loss of generality that $\mathbb{M}=\mathbb{M}^{\prime}$ and $\theta$ is the identity. We must show any $\mathbb{M}_{0}$-module isomorphism $V^{M_{1}} \stackrel{\eta}{\rightarrow} V^{\prime M_{1}}$ extends to an

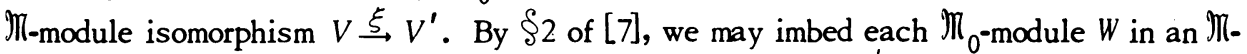
module $W^{*}$ in such a way that any $\Re_{0}$-module isomorphism $W \stackrel{\phi}{\rightarrow} W^{\prime}$ extends to an $\Re_{\text {-module }}$ isomorphism $W^{*} \stackrel{\phi^{*}}{\rightarrow} W^{\prime *}$. But by Theorem 3.1 of [8], we have an M-module isomorphism $V \stackrel{\tau}{\rightarrow}\left(V^{M_{1}}\right)^{*}$ which is the identity on $V^{\pi_{1}}$. Similarly we have an $\mathbb{M}_{\text {-module isomorphism }}$ $V^{\prime} \tau^{\prime}\left(V^{\prime \pi_{1}}\right)^{*}$ which is the identity on $V^{\prime \aleph_{1}}$. Put $\xi=\tau \eta^{*} \tau^{\prime-1}$.

7.3. For the remainder of $\$ 7$, we fix an element $\gamma \in \Pi$. If $\delta \in \Sigma \cup\{0\}$ and $\delta=\Sigma_{\epsilon \in \Pi} n_{\epsilon} \epsilon$, we call $n_{\gamma}$ the $\gamma$-height of $\delta$. Let $m$ be the $\gamma$-height of the highest root of $\Sigma$ and let $\mathcal{L}_{(i)}$ be the sum of the root spaces corresponding to elements of 
$\Sigma \cup\{0\}$ of $\gamma$-height $i, i \in \mathbf{Z}$. Then, $\mathscr{L}_{=} \Sigma_{i=-m}^{m} \mathscr{\complement}_{(i)}$ and $\left[\mathscr{L}_{(i)}, \mathfrak{Q}_{(j)}\right] \subseteq \mathfrak{L}_{(i+j)}$, for $i, j \in \mathbf{Z}$. We have $\mathfrak{L}_{0} \subseteq \mathfrak{L}_{(0)}$ and, if $\Pi \neq\{y\}$, then $\mathfrak{L}_{(0)}=\mathfrak{L}^{P}+\mathfrak{L}_{0}=\mathfrak{L}^{P}+\mathscr{Z}^{P}$, where $P=\Pi-\{y\}$. For $i \in \mathbf{Z}, \mathscr{L}_{(i)}$ is an $\mathfrak{\complement}_{(0)}$-module under the adjoint action.

7.4. Lemma. (i) $\mathfrak{\complement}_{(1)}=\left[\mathscr{\complement}_{(2)}, \mathfrak{L}_{(-1)}\right]$ and $\mathscr{\complement}_{(-1)}=\left[\mathscr{\complement}_{(-2)}, \mathfrak{\complement}_{(1)}\right]$ if $m \geq 2$.

(ii) $\mathfrak{L}_{(2)}=\left[\mathfrak{\complement}_{(1)}, \mathfrak{L}_{(1)}\right]$ and $\mathfrak{\complement}_{(-2)}=\left[\mathfrak{L}_{(-1)}, \mathfrak{L}_{(-1)}\right]$.

(iii) $\mathfrak{L}_{(i)}$ is a completely reducible $\mathfrak{L}_{(0)}$-module for $i \neq 0$.

(iv) $\mathfrak{L}_{(m)}$ is an irreducible $\mathfrak{L}_{(0)}$-module.

(v) The restriction map $\Gamma \rightarrow \Gamma_{(m)}$ is an isomorphism, where $\Gamma_{(m)}$ is the centralizer of ad $\mathscr{\mathscr { L }}_{(m)}\left(\mathscr{L}_{(0)}\right)$ in $\operatorname{End}_{\Phi}\left(\mathscr{\complement}_{(m)}\right)$.

Proof. (i) We prove only the first equality as the second is similar. Let $\delta$ be a root of $\gamma$-height 1 . We must show that $\mathfrak{L}_{\delta} \subseteq\left[\mathscr{L}_{(2)}, \mathfrak{L}_{(-1)}\right]$. Now, $\mathfrak{L}_{(2)} \neq(0)$ and hence there exists a chain $\delta=\gamma_{0}, \gamma_{1}, \cdots, \gamma_{k}$ of elements of $\Sigma$ such that $\gamma_{i}-$ $\gamma_{i-1} \in \Pi$ for $i=1, \cdots, k, \gamma_{i}$ has $\gamma$-height 1 for $i=0, \cdots, k-1$, and $\gamma_{k}$ has $\gamma$ height 2. Now, $\mathfrak{L}_{\gamma_{k-1}}=\left[\mathfrak{L}_{\gamma_{k}}, \mathfrak{L}_{\gamma_{k-1}-\gamma_{k}}\right] \subseteq\left[\mathfrak{L}_{(2)}, \mathfrak{L}_{(-1)}\right]$ and hence we may choose a smallest integer $j \in\{1, \cdots, k-1\}$ such that $\mathfrak{L}_{\gamma} \subseteq\left[\mathfrak{L}_{(2)}, \mathfrak{L}_{(-1)}\right]$. If this integer is not zero, we have $\mathscr{L}_{\gamma_{j-1}}=\left[\mathscr{L}_{\gamma_{j}}, \mathfrak{L}_{\gamma_{j-1}-\gamma_{j}}\right] \subseteq\left[\left[\mathcal{L}_{(2)}, \mathfrak{L}_{(-1)}\right]\right.$, $\left.\mathfrak{L}_{(0)}\right] \subseteq\left[\mathscr{L}_{(2)}, \mathfrak{L}_{(-1)}\right]$ and a contradiction. Hence, $\mathfrak{L}_{\delta}^{j}=\mathscr{L}_{\gamma_{0}}^{j} \subseteq\left[\mathscr{L}_{(2)}, \mathfrak{L}_{(-1)}\right]$.

(ii) is proved similarly.

(iii) It suffices to prove that $\mathscr{Q}$ is a completely reducible $\mathscr{L}(0)^{- \text {module. If }}$ $\Pi=\{\gamma\}, \operatorname{ad}\left(\mathscr{L}_{(0)}\right)=\operatorname{ad}\left(\complement_{0}\right)$ and $\operatorname{ad}\left(\complement_{0}\right)=\operatorname{ad}(\mathcal{C}) \oplus \operatorname{ad}\left(\left[\mathscr{Q}_{0}, \mathfrak{L}_{0}\right]\right)$, where $\operatorname{ad}(\mathcal{C})$ consists of semisimple transformations. If $\Pi \neq\{y\}$ and $P=\Pi-\{y\}$, then $\operatorname{ad}\left(\varrho_{(0)}\right)=$ $\operatorname{ad}\left(\mathcal{Z}^{P}\right) \oplus \operatorname{ad}\left(\mathscr{L}^{P}\right)=\operatorname{ad}\left(\mathcal{Z}^{P} \cap \mathcal{C}\right) \oplus \operatorname{ad}\left(\mathcal{Z}^{P} \cap\left[\mathscr{\complement}_{0}, \mathfrak{L}_{0}\right]\right) \oplus \operatorname{ad}\left(\complement^{P}\right)$, where $\operatorname{ad}(\mathcal{Z} P \cap \mathcal{C})$ consists of semisimple transformations. Thus, in both cases the result is a consequence of Theorem 3.10 of [1].

(iv) is easy to check using 1.4.

(v) By (iv), $\Gamma_{(m)}$ is a division algebra. Thus, the restriction map $\Gamma_{(m)} \rightarrow \Gamma_{\delta}$ is an injection, where $\delta$ is the highest root of $\Sigma$. But by 4.2, the composite map $\Gamma \rightarrow \Gamma_{(m)} \rightarrow \Gamma_{\delta}$ is an isomorphism. Hence, $\Gamma \rightarrow \Gamma_{(m)}$ is an isomorphism.

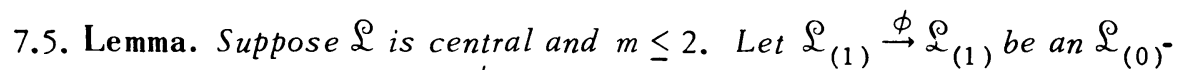
module automorphism such that $\left[L_{1}^{\phi}, L_{1}\right]=0$ for all $L_{1} \in \mathscr{L}_{(1)}$. Then, there exists $a \in \Phi^{*}$ such that $L_{1}^{\phi}=a L_{1}$ for $L_{1} \in \mathscr{L}_{(1)}$.

Proof. By 7.4(v), we may assume $m=2$. Let $\Omega$ be the algebraic closure of $\Phi$. If $\left(\varrho_{(1)}\right)_{\Omega}$ is an irreducible $\left(\varrho_{(0)}\right)_{\Omega}$-module, the result is immediate. Thus, by

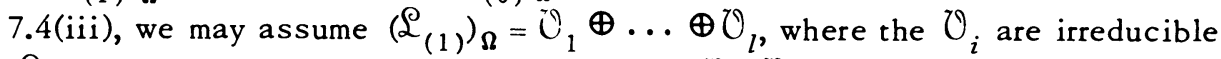
$\left(\complement_{(0)}\right)_{\Omega}$-modules and $l \geq 2$. Now, by $7.4(\mathrm{ii}),\left[\mathcal{O}_{i}, \mathcal{C}_{j}\right] \neq 0$ for some $i, j \in\{1,2, \cdots$, l\}. Thus, $\left[\mathcal{O}_{i}, \mathcal{O}_{j}\right]$ is a nonzero $\left(\bigodot_{(0)}\right)_{\Omega}$-submodule of $\left(\complement_{(m)}\right)_{\Omega}$. Therefore, by 7.4(v), $\left(\bigodot_{(2)}\right)_{\Omega}=\left[\mathcal{O}_{i}, \complement_{j}\right]$. Then, by 7.4(i), we have $\left(\bigodot_{(1)}\right)_{\Omega}=\left[\left(\bigodot_{(2)}\right)_{\Omega},\left(\complement_{(-1)}\right)_{\Omega}\right]=$ $\left[\left[\mathcal{O}_{i}, \mathcal{O}_{j}\right],\left(\mathcal{L}_{(-1)}\right)_{\Omega}\right] \subseteq \mathcal{O}_{i}+\mathcal{O}_{j}$. Thus, since $l \geq 2$, we have $l=2$, and $\{i, j\}=\{1,2\}$. 
Therefore, $\left(\mathcal{C}_{(1)}\right)_{\Omega}=\mathcal{O}_{1} \oplus \mathcal{O}_{2}$ and $\left[\mathcal{O}_{1}, \mathcal{O}_{2}\right] \neq(0)$. Now if $\mathcal{H}$ is a Cartan subalgebra of $\mathcal{Q}$ containing $\mathcal{T}$, then $\mathcal{O}_{1}$ and $\mathcal{O}_{2}$ are each sums of 1 -dimensional root spaces for $\mathcal{H}_{\Omega}$, and hence $\mathcal{O}_{1}$ and $\mathcal{O}_{2}$ are not isomorphic as $\mathcal{H}_{\Omega}$-modules, and therefore they are not isomorphic as $\left(\varrho_{(0)}\right)_{\Omega}$-modules. Thus, $\bigcup_{i}^{\phi}=\bigcup_{i}$ and hence there exists $a_{i} \in \Omega^{*}$ such that $V_{i}^{\phi}=a_{i} V_{i}$ for $V_{i} \in \mathcal{O}_{i}, i=1,2$. Linearizing $\left[L_{1}^{\phi}, L_{1}\right]=0$, we obtain $\left[L_{1}^{\phi}, M_{1}\right]=\left[L_{1}, M_{1}^{\phi}\right]$ for $L_{1}, M_{1} \in \mathscr{L}_{(1)}$. Choosing $V_{1} \in \mathcal{O}_{1}$ and $V_{2} \in \mathcal{O}_{2}$ such that $\left[V_{1}, V_{2}\right] \neq 0$, we have $\left[a_{1} V_{1}, V_{2}\right]=\left[V_{1}, a_{2} V_{2}\right]$ and hence $a_{1}=a_{2}$. Putting $a=a_{1}=a_{2}$, we have $L_{1}^{\phi}=a L_{1}$ for $L_{1} \in \mathfrak{L}_{(1)}$ and hence $a \in \Phi^{*}$.

Suppose $\mathcal{L}^{\prime}$ is another simple algebra and $\gamma^{\prime} \in \Pi^{\prime}$. Define $m^{\prime}$ and $\mathscr{L}_{(i)}^{\prime}(i \in \mathbf{Z})$ as in 7.3 .

7.6. Proposition. Suppose $\mathcal{Q}$ and $\mathfrak{L}^{\prime}$ are central and $m=m^{\prime} \leq 2$. Suppose there exists an isomorphism $\mathscr{L}_{(0)} \stackrel{\theta_{0}}{\longrightarrow} \mathscr{L}_{(0)}^{\prime}$ and suppose there exist $\theta_{0}$-equivalences $\mathcal{L}_{(i)} \stackrel{\theta_{i}}{\longrightarrow} \mathfrak{L}_{(i)}^{\prime}, i=1,2$.

Then, there exists an isomorphism $\stackrel{\varrho}{\rightarrow} \bigodot^{\prime}$ and $a \in \Phi^{*}$ such that $\theta \mid \complement_{(0)}=\theta_{0}$, $\theta \mid £_{(1)}=\theta_{1}$, and $\theta \mid £_{(2)}=a \theta_{2}$.

Proof. In this proof, $L_{i}$ and $M_{i}$ will denote arbitrary elements of $\mathscr{L}_{(i)}$ for $i \in \mathbf{Z}$. Define $\mathscr{L}_{(-i)} \stackrel{\theta_{-i}}{\rightarrow} \mathfrak{L}_{(-i)}^{\prime}$ so that $\left(L_{i}^{\theta_{i}}, L_{-i}^{\theta_{-i}}\right)=\left(L_{i}, L_{-i}\right), i=1,2$. (Here $($, denotes both Killing forms.) Then, $\left(\left[L_{-i}, L_{0}\right]^{\theta}-i, L_{i}^{\theta_{i}}\right)=\left(\left[L_{-i}, L_{0}\right], L_{i}\right)=$ $\left(L_{-i}\left[L_{0}, L_{i}\right]\right)=\left(L_{-i}^{\theta},\left[L_{0}, L_{i}\right]^{\theta_{i}}\right)=\left(L_{-1}^{\theta_{-i}^{i}},\left[L_{0}^{\theta_{0}}, L_{i}^{\theta_{i}}\right]\right)=\left(\left[L_{-i}^{\theta_{-i}}, L_{0}^{\theta_{0}}\right], L_{i}^{\theta_{i}}\right)$ and hence $\left[L_{-i}, L_{0}\right]^{\theta_{-i}}=\left[L_{-i}^{\theta_{-}}, L_{0}^{\theta_{0}}\right], i=1$, 2. (Subsequent identities have similar proofs and so we omit their verifications.) This combined with our assumptions gives $\left[L_{i}, L_{0}\right]^{\theta_{i}}=\left[L_{i}^{\theta_{i}}, L_{0}^{\theta_{0}}\right],-2 \leq i \leq 2$. Therefore, $\left(L_{0}^{\theta_{0}}, M_{0}^{\theta_{0}}\right)=\left(L_{0}, M_{0}\right)$. Hence, one easily checks that $\left[L_{i}, L_{-i}\right]^{\theta_{0}}=\left[L_{i}^{\theta_{i}}, L_{-i}^{\theta_{-i}}\right], i=0,1$, 2. If $m=m^{\prime}=1$, we may define $\theta$ by $\theta \mid \mathscr{L}_{(i)}=\theta_{i}, i=-1,0,1$. Suppose then that $m=m^{\prime}=2$.

Put $\mathfrak{L}^{(2)}=\mathfrak{L}_{(-2)} \oplus \mathfrak{L}_{(0)} \oplus \mathfrak{L}_{(2)}$ and $\mathcal{O}=\mathfrak{L}_{(1)} \oplus \mathfrak{L}_{(-1)} \cdot \mathfrak{L}^{(2)}$ is a Lie algebra with decomposition $\left(\mathscr{L}_{(-2)}, \mathscr{L}_{(0)}, \mathfrak{L}_{(2)}\right) ; \mathcal{O}$ is an irreducible $\mathscr{L}^{(2)}$-module; and the set of elements of $\mathcal{O}$ annihilated by $\mathscr{L}_{(2)}$ is $\mathscr{L}_{(1)}$. We have similar definitions and remarks for $\mathscr{L}^{\prime(2)}$ and $\mathcal{O}^{\prime}$. Define $\mathscr{L}^{(2)} \stackrel{\theta(2)}{\rightarrow}^{\prime \prime(2)}$ by putting $\theta^{(2)} \mid \mathscr{L}_{(i)}=\theta_{i}, i=-2$, 0 , 2. By the previous paragraph, $\theta^{(2)}$ is a Lie isomorphism. By 7.2 , the $\theta_{0}$-equivalence $\mathscr{L}_{(1)} \stackrel{\theta_{1}}{\rightarrow} \mathfrak{Q}_{(1)}^{\prime}$ extends to a $\theta^{(2)}$ equivalence $\mathcal{O} \stackrel{\xi}{\rightarrow} 7 \mathcal{O}^{\prime}$. Thus, by $7.4(\mathrm{i})$, $\mathfrak{L}_{(-1)}^{\xi}=\left[\mathscr{L}_{(1)}, \mathfrak{L}_{(-2)}\right]^{\xi}=\left[\mathscr{\complement}_{(1)}^{\theta_{1}}, \mathfrak{L}_{(-2)}^{\theta}-2\right]=\left[\mathfrak{Q}_{(1)}^{\prime} \mathfrak{\complement}_{(-2)}^{\prime}\right]=\mathfrak{L}_{(-1)}^{\prime}$. Define $\mathfrak{L}_{(-1)} \stackrel{\xi-1}{\longrightarrow}$ $\mathfrak{Q}_{(-1)}^{\prime}$ by $\xi_{-1}=\xi \mid \complement_{(-1)}$. Then, $\left[L_{1}, L_{-2}\right]^{\xi}-1=\left[L_{1}^{\theta_{1}}, L_{-2}^{\theta_{-2}^{2}}\right.$ and $\left[L_{-1}, L_{2}\right]^{\theta_{1}}=$ $\left[L^{\xi}-1, L_{2}^{\theta_{2}^{2}}\right]$. As a consequence, it is easy to check that $\left[L_{-1}, M_{-1}\right]^{\theta}-2=$ $\left[L_{-1}^{\theta}-1, M_{-1}^{\xi}\right]$ and hence $\left[L_{-1}^{\xi}-1^{\theta} \theta_{-1}^{-1}, L_{-1}\right]=0$. Then, by 7.5 , there exists $a \in \Phi^{*}$ such that $L_{-1}^{\xi}=a L_{-1}^{\theta}$. Hence, $\left[L_{1}, L_{-2}\right]^{\theta}-1=\left[L_{1}^{\theta_{1}}, a^{-1} L_{-2}^{\theta}\right],\left[L_{-1}, L_{2}\right]^{\theta_{1}}=$ $\left[L_{-1}^{\theta}, a L_{2}^{\theta_{2}}\right]$, and $a^{-1}\left[L_{-1}, M_{-1}\right]^{\theta_{-2}}=\left[L_{-1}^{\theta}, M_{-1}^{\theta}\right]$. One checks as a result that $a\left[L L_{1}, M_{1}\right]^{\theta_{2}}=\left[L_{1}^{\theta_{1}}, M_{1}^{\theta_{1}}\right]$. These last four identites, together with the first 
paragraph, tell us that the map $\stackrel{\varrho}{\rightarrow} \mathcal{L}^{\prime}$ defined by $\theta\left|\mathscr{L}_{(i)}=\theta_{i}, i=-1,0,1, \theta\right| \mathscr{L}_{(2)}=$ $a \theta_{2}$, and $\theta \mid £_{(-2)}=a^{-1} \theta_{-2}$, is a Lie isomorphism.

7.7. Corollary. Suppose $\mathcal{L}_{\text {and }} \mathscr{L}^{\prime}$ are central and $m=m^{\prime}=1$. Then, any $\theta_{0^{-}}$ equivalence $\mathfrak{L}_{(1)} \rightarrow \mathfrak{L}_{(1)}^{\prime}$ extends to an isomorphism $\mathfrak{L}^{\rightarrow} \mathfrak{L}^{\prime}$.

8. Compatibility. We assume in this section that $\mathcal{L}^{\prime}$ is another simple Lie algebra and we assume there exists some fixed isomorphism $\gamma \rightarrow \gamma^{\prime}$ of $\Sigma$ onto $\Sigma^{\prime}$, i.e., $\gamma \rightarrow \gamma^{\prime}$ is a bijection such that if $\gamma, \delta \in \Sigma$, then $\gamma+\delta \in \Sigma$ if and only if $\gamma^{\prime}+\delta^{\prime} \in \Sigma^{\prime}$, in which case $(\gamma+\delta)^{\prime}=\gamma^{\prime}+\delta^{\prime}$. The map $\gamma \rightarrow \gamma^{\prime}$ induces a group isomorphism $w^{2} \rightarrow w^{\prime}$ of 20 onto $60^{\prime}$ such that $\left(w_{\gamma}\right)^{\prime}=w^{2} \gamma^{\prime}$ and $\left(\gamma^{w 2}\right)^{\prime}=\gamma^{\prime} \mathcal{w}^{\prime}$ for $\gamma \in \Sigma$ and $w \in W$.

8.1. Proposition. Let $(\varrho, \mathcal{T}) \stackrel{\theta}{\rightarrow}\left(\varrho^{\prime}, \mathcal{J}^{\prime}\right)$ be an isomorphism which induces $\gamma \rightarrow \gamma^{\prime}$ on the root systems (i.e. $T_{\gamma}^{\theta}=T_{\gamma^{\prime}}$ for $y \in \Sigma$ ). Let $\theta_{0}=\theta \mid \&_{0}$. Then, $\theta_{0} \phi\left(w^{\prime}\right) \theta_{0}^{-1} \phi(w)^{-1}$ is inner on $\mathfrak{Q}_{0}$ for $w \in \mathcal{W}$.

Proof. For $\gamma \in \Sigma$, we have $T_{\gamma}^{\theta}{ }^{\phi\left(\mathcal{L}^{\prime}\right)}=T_{\gamma^{\prime}}^{\phi\left(\mathcal{w}^{\prime}\right)}=T_{\left(\gamma^{\mathcal{N}}\right)^{\prime}}=T_{\gamma}^{\phi(w) \theta_{0}}$ and hence $\theta_{0} \phi\left(w^{\prime}\right) \theta_{0}^{-1} \phi(w)^{-1}$ fixes the elements of $\mathfrak{T}$. The result follows now from 2.5 .

8.2. The proposition motivates us to make the following definition. Let $\left(\bigodot_{0}\right.$, T) $\stackrel{\theta_{0}}{\rightarrow}\left(\mathscr{Q}_{0}^{\prime}, \mathfrak{T}^{\prime}\right)$ be an isomorphism which induces $\gamma \rightarrow \gamma^{\prime}$ on the root systems. For $w \in \mathbb{W}$, we say $\theta_{0}$ is compatible with $w$ if $\theta_{0} \phi\left(w^{\prime}\right) \theta_{0}^{-1} \phi(w)^{-1}$ is inner on $\mathfrak{L}_{0}$. If $w=w_{y}$, we say $\theta_{0}$ is compatible with $\gamma$.

The choice of $\phi(w)$ and $\phi\left(w^{\prime}\right)$ is by no means unique. Nevertheless, compatibility is well defined. For if $\phi_{1}(w)$ and $\phi_{1}\left(w^{\prime}\right)$ are other choices of $\phi(w)$ and $\phi\left(w^{\prime}\right)$ (resp.) satisfying 5.1 , then

$$
\theta_{0} \phi_{1}\left(w^{\prime}\right) \theta_{0}^{-1} \phi_{1}(w)^{-1}=\left(\theta_{0} \phi_{1}\left(w^{\prime}\right) \phi\left(w^{\prime}\right)^{-1} \theta_{0}^{-1}\right)\left(\theta_{0} \phi\left(w^{\prime}\right) \theta_{0}^{-1} \phi(w)^{-1}\right)\left(\phi(w) \phi_{1}(w)^{-1}\right) \text {. }
$$

By 2.5, $\theta_{0} \phi_{1}\left(w^{\prime}\right) \phi\left(w^{\prime}\right)^{-1} \theta_{0}^{-1}$ and $\phi(w) \phi_{1}(w)^{-1}$ are inner on $\mathfrak{L}_{0}$. Hence, $\theta_{0} \phi\left(w^{\prime}\right) \theta_{0}^{-1} \phi(w)^{-1}$ is inner on $\mathfrak{Q}_{0}$ if and only if $\theta_{0} \phi_{1}\left(w^{\prime}\right) \theta_{0}^{-1} \phi_{1}(w)^{-1}$ is inner on $\mathfrak{L}_{0}$. I.e., compatibility is well defined.

In order to check that $\theta_{0}$ is compatible with $w^{2}=w_{1} w_{2}$, it suffices to check that $\theta_{0}$ is compatible with $w_{1}$ and with $w_{2}$, where $w_{1}, w_{2} \in \mathcal{W}$. This remark follows from the identity $\theta_{0} \phi\left(w_{1}^{\prime}\right) \phi\left(w_{2}^{\prime}\right) \theta_{0}^{-1}\left(\phi\left(w_{1}\right) \phi\left(w_{2}\right)\right)^{-1}=\psi_{1} \phi\left(w_{1}\right) \psi_{2} \phi\left(w_{1}\right)^{-1}$, where $\psi_{i}=\theta_{0} \phi\left(w_{i}^{\prime}\right) \theta_{0}^{-1} \phi\left(w_{i}\right)^{-1}, i=1,2$, and the fact that we may choose $\phi\left(w_{1} w_{2}\right)=$ $\phi\left(w_{1}\right) \phi\left(w_{2}\right)$ and $\phi\left(w_{1}^{\prime} w_{2}^{\prime}\right)=\phi\left(w_{1}^{\prime}\right) \phi\left(w_{2}^{\prime}\right)$. Thus, if $\theta_{0}$ is compatible with all $\gamma \in \Pi$, it is compatible with all $w \in \mathcal{W}$.

For the remainder of this section, we assume $\left(\complement_{0}, \mathfrak{T}\right) \stackrel{\theta_{0}}{\rightarrow}\left(\varrho_{0}^{\prime}, \mathcal{J}^{\prime}\right)$ is an isomorphism which induces $\gamma \rightarrow \gamma^{\prime}$ on the root systems.

8.3. Lemma. Suppose $\theta_{0}$ is compatible with all $w \in W$. Suppose $\delta \in \Sigma$ and 
there exists a $\theta_{0}$-equivalence $\mathfrak{L}_{\delta} \stackrel{\theta_{\delta}}{\longrightarrow} \mathscr{Q}_{\delta^{\prime}}^{\prime}$. Then, for any $\gamma \in \Sigma$ conjugate to $\delta$ under 6 , we bave

(i) $\left(Z^{\gamma}\right)^{\theta_{0}}=Z^{\gamma^{\prime}}$,

(ii) $\left(\varrho_{0}^{\gamma}\right)^{\theta_{0}}=\mathscr{L}_{0}^{\prime} \gamma^{\prime}$, provided that $2 \delta \notin \Sigma$,

(iii) There exists a $\theta_{0}$-equivalence $\mathfrak{L}_{\gamma} \stackrel{\theta_{\gamma}}{\longrightarrow} \mathfrak{L}_{\gamma}^{\prime}$, provided that $\mathfrak{Q}$ is central and $\delta$ is conjugate to the bighest root of $\Sigma$ under $(0$.

Proof. (i) Certainly, $\left(Z^{\delta}\right)^{\theta_{0}}=Z^{\delta^{\prime}}$. But for $w \in \mathcal{W}, \theta_{0} \phi\left(w^{\prime}\right) \theta_{0}^{-1} \phi(w)^{-1}$ stabilizes $\mathscr{Z}^{\delta}$ (by 2.4) and hence $\left.\left(\mathcal{Z}^{\delta \omega}\right)^{\theta_{0}}=\mathscr{Z}^{(\delta \omega}\right)^{\prime}$ for all $w$.

(ii) As in (i), it suffices to show that $\left(\mathcal{Q}_{0}^{\delta}\right)^{\theta_{0}^{-}}=\mathfrak{L}_{0}^{\prime} \delta^{\prime}$. But $\mathfrak{L}_{0}^{\delta}=\left(\mathcal{C} \cap \mathfrak{L}_{0}^{\delta}\right) \oplus$ $\mathfrak{Q}^{\delta}$ and $\mathcal{L}_{0}^{\prime} \delta^{\prime}=\left(\mathcal{C}^{\prime} \cap \mathfrak{L}_{0}^{\prime} \delta^{\prime}\right) \oplus \mathfrak{A}^{\delta^{\prime}}$. Since $\mathfrak{Q}^{\delta}$ is the sum of the simple summands of $\left[\mathfrak{Q}_{0}, \mathfrak{L}_{0}\right]$ which act nontrivially on $\mathfrak{L}_{\delta}$, we have $\left(\mathfrak{Q}^{\delta}\right)^{\theta_{0}}=\mathfrak{Q}^{\delta^{\prime}}$. Also, $\mathfrak{C}^{\theta_{0}}=$ $\mathcal{C}^{\prime}$. Hence, by 5.5 , we need only show that for $L_{0} \in \mathcal{C}$, we have $L_{0}^{\phi(w \delta)}=-L_{0}$ if and only if $L_{0}^{\theta_{0} \phi\left(w_{\delta}\right)}=-L_{0}^{\theta_{0}}$. This however follows from the fact that $\theta_{0} \phi\left(\omega_{\delta}{ }^{\prime}\right) \theta_{0}^{-1} \phi\left(\omega_{\delta}\right)^{-1}$ fixes every element of $\mathcal{C}$.

(iii) Choose $w \in \mathcal{W}$ such that $\delta=\gamma^{w}$. Let $\psi=\theta_{0} \phi\left(w^{\prime}\right) \theta_{0}^{-1} \phi(w)^{-1}$. By 2.4 and 4.3 , there exists an invertible $\eta \in$ End $_{\Phi}\left(\mathscr{Q}_{\gamma}\right)$ such that $\eta^{-1}$ ad $\rho_{\gamma}\left(L_{0}\right) \eta=$ $\operatorname{ad}_{\mathcal{P}_{\gamma}}\left(L_{0}^{\psi}\right)$. It is easily checked then that if $\mathcal{L}_{\gamma} \stackrel{\theta_{\gamma}}{\longrightarrow} \mathcal{L}_{\gamma}^{\prime}$, is defined by $\theta_{\gamma}=$ $\eta \phi(w) \theta_{\delta} \phi\left(w^{\prime}\right)^{-1}$, we have

$$
\theta_{\gamma}^{-1} \operatorname{ad}_{\mathscr{L}_{\gamma}}\left(L_{0}\right) \theta_{\gamma}=\operatorname{ad}_{\mathfrak{L}_{\gamma^{\prime}}^{\prime}}\left(L_{0}^{\theta_{0}}\right)
$$

i.e. $\theta_{y}$ is a $\theta_{0}$-equivalence.

8.4. Lemma. Suppose $\theta_{0}$ is compatible with all $w \in \mathcal{W}$ and suppose all the roots of $\Sigma$ are conjugate under 6 . Suppose for some $\delta \in \Sigma$, there exists a $\theta_{0^{-}}$ equivalence $\mathfrak{L}_{\delta} \stackrel{\theta_{\delta}}{\rightarrow} \mathfrak{\complement}_{\delta}^{\prime}$. Then, we may identify the centrum $\Gamma$ of $\mathfrak{L}$ with the centrum $\Gamma^{\prime}$ of $\mathcal{L}^{\prime}$ in sucb a way that $\theta_{0}$ and $\theta_{\delta}$ are $\Gamma$-linear.

Proof. By 4.2, we have an isomorphism $\Gamma \rightarrow \Gamma^{\prime}$ which is the composition of the maps $\Gamma \rightarrow \Gamma_{\delta} \rightarrow \Gamma_{\delta}{ }^{\prime} \rightarrow \Gamma^{\prime}$, where the first map is the restriction map, the second map is conjugation by $\theta_{\delta}$, and the third map is the inverse of the restriction map. If we use this isomorphism $\Gamma \rightarrow \Gamma^{\prime}$ to identify $\Gamma$ and $\Gamma^{\prime}$, it is easy to check that $\left(c L_{\delta}\right)^{\theta_{\delta}}=c L_{\delta}^{\theta_{\delta}}$ for $c \in \Gamma$ and $L_{\delta} \in \mathcal{L}_{\delta}$. It remains to check that $\left(c L_{0}\right)^{\theta_{0}}=c L_{0}^{\theta_{0}}$ for $L_{0} \in \mathscr{L}_{0}$.

Let $c \in \Gamma$ and $L_{0} \in \mathfrak{L}_{0}$. Then, for $L_{\delta} \in \mathfrak{L}_{\delta}$, we have $\left[L_{\delta}^{\theta_{\delta}},\left(c L_{0}\right)^{\theta_{0}}\right]=$ $\left[L_{\delta}, c L_{0}\right]^{\theta_{\delta}}=\left[c L_{\delta}, L_{0}\right]^{\theta_{\delta}}=\left[\left(c L_{\delta}\right)^{\theta_{\delta}}, L_{0}^{\theta_{0}}\right]=\left[c L_{\delta}^{\theta_{\delta}}, L_{0}^{\theta_{0}}\right]=\left[L_{\delta}^{\theta_{\delta}}, c L_{0}^{\theta_{0}}\right]$ and hence $c L_{0}^{\theta_{0}}-\left(c L_{0}\right)^{\theta_{0}} \in \mathscr{Z}^{\delta^{\prime}}$. Thus, for $w=\mathcal{W}^{0}, \mathcal{Z}^{\left(\delta \omega^{2}\right)^{\prime}}$ contains $\left(c L_{0}^{\theta_{0}}-\left(c L_{0}\right)^{\theta_{0}}\right)^{\phi\left(\mathcal{N}^{\prime}\right)}=c L_{0}^{\theta_{0} \phi\left(\mathcal{N}^{\prime}\right)}-\left(c L_{0}\right)^{\theta_{0} \phi\left(\omega^{\prime}\right)}=c L_{0}^{\psi \phi(w) \theta_{0}}-\left(c L_{0}\right)^{\psi \phi(\mathcal{N}) \theta_{0}}=$ $c\left(L_{0}^{\psi \phi(w)}\right)^{\theta_{0}}-\left(c L_{0}^{\psi \phi(w)}\right)^{\theta_{0}}$, where $\psi=\theta_{0} \phi\left(w^{\prime}\right) \theta_{0}^{-1} \phi(w)^{-1}$. Therefore, since 
$\mathscr{L}_{0}^{\psi \phi(w)}=\mathscr{L}_{0}$, we have $c L_{0}^{\theta_{0}}-\left(c L_{0}\right)^{\theta_{0}} \in \mathcal{Z}^{\left(\delta \delta^{2}\right)^{\prime}}$ for all $w \in \mathcal{W}$, in which case $c L_{0}^{\theta_{0}}=\left(c L_{0}\right)^{\theta_{0}}$, since $\delta^{\omega \nu}$ runs over all elements of $\Sigma$.

9. The isomorphism theorem. Suppose $\mathcal{L}$ and $\mathcal{L}^{\prime}$ are central simple Lie algebras. Let $\left\{\delta_{1}, \cdots, \delta_{k}\right\}$ be a set of elements of $\Sigma$ which represents all root lengths of $\Sigma$ exactly once. (Thus, $k=1$ if $\Pi$ is of type $A, D$, or $E ; k=2$ if $\Pi$ is of type $B, C, F, G$, or $B C_{1}$; and $k=3$ if $\Pi$ is of type $B C_{r}(r \geq 2)$.) We assume $\delta_{k}$ is of maximum length.

9.1. Theorem. Suppose $\left(\varrho_{0}, \mathcal{T}\right) \stackrel{\theta_{0}}{\longrightarrow}\left(\varrho_{0}^{\prime}, \mathcal{J}^{\prime}\right)$ is an isomorphism which induces an isomorphism $\gamma \rightarrow \gamma^{\prime}$ of $\Sigma$ onto $\Sigma^{\prime}$ and which is compatible with all $\gamma \in \Pi$. Suppose there exist $\theta_{0}$-equivalences $\mathcal{L}_{\delta_{i}} \stackrel{\theta_{\delta_{i}}}{\longrightarrow} \mathfrak{\varrho}_{\delta_{i}}^{\prime}, i=1, \cdots, k$. Then, there exists an isomorphism $\stackrel{\varrho}{\rightarrow} \mathfrak{\complement}^{\prime}$ which extends $\theta_{0}$ and which extends $\theta_{\delta_{i}}$ if $i=1$ or $i \neq k$.

Proof. We may assume $r>0$. The proof is by induction on the rank $r$ of $\mathfrak{Q}$. If $r=1$, the theorem is a consequence of 7.6. Suppose $r \geq 2$. By 6.6 and the classical result, we may assume $\Pi$ is not of type $D$ or $E$.

We consider the reduced case first. Label the roots of $\Pi$ as $\gamma_{1}, \gamma_{2}, \cdots, \gamma_{r}$, where $\gamma_{i}$ is connected to $\gamma_{i+1}(i=1, \cdots, r-1)$ and $\gamma_{r}$ is of maximum length. Put $P=\left\{\gamma_{1}, \cdots, \gamma_{r-1}\right\}$. Rechoosing $\Pi$, we may assume the $\delta_{i}$ of minimum length, $\delta_{1}$ say, is contained in $\langle P\rangle$. By $8.3\left((\mathrm{i})\right.$ and (ii)), $\left(\mathcal{L}^{P}\right)^{\theta_{0}}=\mathcal{Z}^{i} P^{\prime}$ and $\left(\mathscr{C}_{0}^{P}\right)^{\theta_{0}}=\mathscr{L}_{0}^{\prime} P^{\prime}$. Then, $\theta_{0} \mathfrak{L}_{0}^{P}$ and $\theta_{\delta_{1}}$ extend to an isomorphism $\mathscr{L}^{P} \stackrel{\theta^{P}}{\rightarrow} \mathcal{L}^{\prime} P^{\prime}$. (To see this, one considers two cases. If $\Pi$ is of type $B_{r}(r \geq 3)$ or $F_{4}$, then $\langle P\rangle$ contains two root lengths and the statement follows from 8.3(iii), 4.3, and the induction hypothesis. On the other hand if $\Pi$ is of type $C_{r}(r \geq 2), A_{r}(r \geq 2)$, or $G_{2}$, then $\langle P\rangle$ contains one root length and the statement follows from 8.4 and the induction hypothesis.) Let $\mathfrak{L}_{(i)}(i \in \mathbf{Z})$ and $m \in \mathbf{Z}$ be defined as in 7.3 , using the root $\gamma_{r}$. Define $\mathscr{L}_{(i)}^{\prime}$ $(i \in \mathbf{Z})$ and $m^{\prime}$ similarly. Then, $1 \leq m=m^{\prime} \leq 2$. We define an isomorphism $\mathscr{L}_{(0)}=$ $\mathcal{L}^{P} \oplus \mathcal{Z}^{P \theta_{(0)}} \mathfrak{L}_{(0)}^{\prime}=\mathcal{L}^{\prime} P^{\prime} \oplus \mathscr{Z}^{P^{\prime}}$ by putting $\left.\theta_{(0)}\right|^{P P}=\theta^{P}$ and $\theta_{(0)}\left|\mathcal{Z}^{P}=\theta_{0}\right| \mathcal{Z}^{P}$. By 7.6, it suffices to find $\theta_{(0)}$-equivalences $\mathcal{L}_{(i)} \stackrel{\theta_{(i)}}{\longrightarrow} \mathcal{Q}_{(i)}^{\prime}, i=1, \cdots, m$. But $\left(\Sigma_{\gamma \in\langle P\rangle}-\mathscr{L}_{\gamma}, \mathfrak{L}_{0}, \Sigma_{\gamma \in(P\rangle}+\mathfrak{L}_{\gamma}\right)$ is a decomposition for $\mathscr{L}_{(0)}$, and the submodule of $\mathscr{L}_{(m)}$ annihilated by $\Sigma_{\gamma \in\langle P\rangle^{+}} \mathcal{L}_{\gamma}$ is $\mathcal{L}_{\epsilon}$ where $\epsilon$ is the highest root of $\Sigma$. Moreover, by 8.3 (iii), we have a $\theta_{0}$-equivalence, $\mathscr{L}_{\epsilon} \rightarrow \mathfrak{L}_{\epsilon}^{\prime}$. Hence by 7.2 , there is a $\theta_{(0)}$-equivalence $\mathfrak{L}_{(m)} \rightarrow \mathfrak{L}_{(m)}^{\prime}$. It remains to find $\theta_{(1)}$ when $m=2$. In this case $\mathcal{L}$ is of type $G_{2}$ or $F_{4}$. Put $\lambda=3 \gamma_{1}+\gamma_{2}$ in the first case and $\lambda=2 \gamma_{1}+4 \gamma_{2}+3 \gamma_{3}+$ $\gamma_{4}$ in the second. By 8.3(iii), we have a $\theta_{0}$-equivalence $\mathfrak{L}_{\lambda} \rightarrow \mathfrak{Q}_{\lambda^{\prime}}^{\prime}$, which ex-

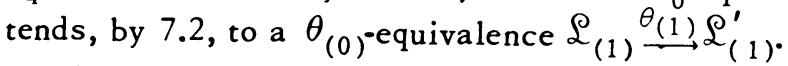

We turn now to the nonreduced case. The roots of $\Pi$ may be labelled so that II has Dynkin diagram: 


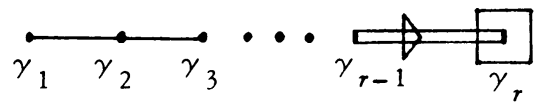

Put $P=\left\{\gamma_{1}, \cdots, \gamma_{r-1}\right\}$. Rechoosing $\Pi$ and relabelling the $\delta_{i}$, we may assume $\delta_{1} \in\langle P\rangle, \delta_{2}=\gamma_{1}+\gamma_{2}+\cdots+\gamma_{r-1}+\gamma_{r}$, and $\delta_{3}$ is a root of maximum length. Define $\mathcal{L}_{(i)}(i \in \mathbf{Z})$, and $\mathcal{L}_{(i)}^{\prime}(i \in \mathbf{Z})$ as in the reduced case. As in the reduced case, we may extend $\theta_{0}$ and $\theta_{\delta_{1}}$ to an isomorphism $\mathcal{L}_{(0)} \stackrel{\theta_{(0)}}{\longrightarrow} \mathcal{Q}^{\prime}(0)$. Once again we look for $\theta_{(0)}$-equivalences $\theta_{(1)}$ and $\theta_{(2)}$, this time requiring that $\theta_{(1)}$ extends $\theta_{\delta_{2}}$. $\theta_{(2)}$ is found exactly as in the reduced case. Finally, since $\mathscr{L}_{\delta_{2}}$ is the submodule of $\mathcal{L}_{(1)}$ annihilated by $\Sigma_{y \in\langle P\rangle}+\mathfrak{L}_{\gamma}$, we have the required $\theta_{(0)}$-equivalence $\theta_{(1)}$ extending $\theta_{\delta_{2}}$ by 7.2 .

9.2. If $k=2$ or 3 , the theorem does not guarantee, that $\theta$ extends $\theta_{\delta_{k}}$. However, we note that $\left(\theta \mid \mathscr{L}_{\delta_{k}}\right) \theta_{\delta_{k}}^{-1}$ commutes with the adjoint action of $\mathfrak{L}_{0}$ on $\mathcal{L}_{\delta_{k}}$ and hence, by $4.2, \theta \mid \mathcal{L}_{\delta_{k}}$ and $\theta_{\delta_{k}}$ differ by at most an element of $\Phi^{*}$.

9.3. We now give an example to show that the $\theta_{0}$-equivalences $\theta_{\delta_{i}}$ cannot be removed from the hypothesis of 9.1 . Let $\Delta$ be a central division algebra of degree $d>1$ over $\Phi$. Suppose $J$ (resp. $J^{\prime}$ ) is an involution of $\Delta$ of the first kind such that the skew elements of $\Delta$ relative to $J$ (resp. $J^{\prime}$ ) have dimension $1 / 2 d(d-1$ ) (resp. $1 / 2 d(d+1))$. Let $\mathcal{L}\left(\right.$ resp. $\left.\mathcal{L}^{\prime}\right)$ be the Lie algebra of skew transformations with respect to a $J$-hermitian (resp. $J$ '-hermitian) form of index $r$, on a space of dimension $2 r$ over $\Delta$. Then, $\mathscr{L}$ and $\mathcal{L}$ are central simple with root systems of type $C_{r} \cdot \mathscr{L}_{0}$ and $\mathcal{L}_{0}^{\prime}$ are both isomorphic to $r$ copies of $\Delta$. In fact there is an isomorphism $\gamma \rightarrow$ $\gamma^{\prime}$ of $\Sigma$ onto $\Sigma^{\prime}$ and an isomorphism $\left(\varrho_{0}, \mathcal{T}\right) \stackrel{\theta_{0}}{\rightarrow}\left(\varrho_{0}^{\prime}, \mathcal{J}^{\prime}\right)$ inducing $\gamma \rightarrow \gamma^{\prime}$ and compatible with all $w \in W$. Moreover, if $\gamma$ is a short root of $\Sigma$, these maps can be chosen so that there is a $\theta_{0}$-equivalence $\mathcal{L}_{\gamma} \stackrel{\theta}{\gamma}_{\longrightarrow} \mathcal{Q}_{\gamma^{\prime}}^{\prime}$. However, if $\delta$ is a long root, $\mathfrak{L}_{\delta}$ and $\mathfrak{L}_{\delta}^{\prime}$, have different dimensions (namely $1 / 2 d(d-1)$ and $1 / 2 d(d+1$ ) respectively). Therefore, $\mathcal{L}$ and $\mathcal{L}^{\prime}$ are not isomorphic.

In concluding, the author would like to express his gratitude to Professor G. B. Seligman for suggesting the isomorphism problem and for his helpful comments.

\section{REFERENCES}

1. N. Jacobson, Lie algebras, Interscience Tracts in Pure and Appl. Math., no. 10, Interscience, New York, 1962. MR $26 \# 1345$.

2. —- A note on automorphisms of Lie algebras, Pacific J. Math. 12 (1962), 303-315. MR $26 \# 6222$.

3. I. Satake, On the theory of reductive algebraic groups over a perfect field, J. Math. Soc. Japan 15 (1963), 210-235. MR $27 \# 1438$.

4. G. Seligman, Topics in Lie algebras, Mimeographed notes, Yale University, New Haven, Conn., 1969.

5. J.-P. Serre, Algèbres de Lie semi-simples complexes, Benjamin, New York, 1966. MR $35 \# 6721$. 
6. J. Tits, Classification of algebraic semis imple groups, Proc. Sympos. Pure Math., vol. 9, Amer. Math. Soc., Providence, R. I., 1966, pp. 33-62. MR 37 \#309.

7. N. Wallach, Induced representations of Lie algebras and a theorem of Borel-Weil, Trans. Amer. Math. Soc. 136 (1969), 181-187. MR 38 \#2258.

8. - Induced re presentations of Lie algebras. II, Proc. Amer. Math. Soc. 21 (1969), 161-166. MR 38 \#5871.

DEPARTMENT OF MATHEMATICS, UNIVERSITY OF ALBERTA, EDMONTON, ALBERTA, CANADA 